\title{
Profilo terapeutico e farmacoeconomico di linezolid
}

Mario Eandi*

PROFILO

\begin{abstract}
The incidence of nosocomial infections from Gram-positive pathogens has been increasing in the last two decades, alongside the development of antibiotic resistance in many bacteria. Glycopeptidic drugs are the most widely used options for these patients, but some bacterial strains with low sensibility to vancomycin and teicoplanin are starting to emerge, warranting careful monitoring and control of nosocomial and also community-acquired infections.
\end{abstract}

This paper outlines a clinical, therapeutic and economic profile of linezolid, the first drug of the only new antibiotic class developed in the last thirty years. In clinical trials, linezolid has demonstrated very promising efficacy and safety in the treatment of antibiotic-resistant infections, in particular those caused by methicillin-resistant staphylococci (MRSA), obtaining greater or equal clinical and microbiological success rates than the standard options. Linezolid, as most newer drugs, has higher acquisition costs than the alternatives, but also bears interesting features that may modify the formation of infection treatment costs. In particluar, linezolid is very well absorbed after oral administration, allowing the planning of sequential iv/os strategies that have the potential to reduce health care costs and to improve the quality of life of the patients by shortening the length of hospitalization.

Economic evaluations have demonstrated that this advantage is not merely theoretical, but that it can be achieved in real practice. In particular, linezolid has been shown to be more cost-effective than teicoplanin and vancomycin in the treatment of hospitalized, MRSA-related nosocomial pneumonia and severe infections.

Farmeconomia e percorsi terapeutici 2004; 5 (2): 81 -102

\section{INTRODUZIONE}

L'incidenza di gravi infezioni sostenute da patogeni Gram-positivi è aumentata in modo preoccupante negli ultimi due decenni, soprattutto tra i pazienti ricoverati in ospedale [1-3]. Parallelamente a questo fenomeno, soprattutto nelle corsie degli ospedali, sono comparsi con sempre maggior frequenza ceppi di Gram-positivi resistenti a uno o più antibiotici, come i ceppi di Staphylococcus aureus meticillino-resistenti (MRSA) o multiresistenti, i ceppi di Streptococcus pneumoniae penicillino- ed eritromicina-resistenti e, negli ultimi anni, anche i ceppi di enterococchi e di stafilococchi vancomicina- e/o teicoplanina-resistenti [4-8].

I glicopeptidi, vancomicina e teicoplanina, sono antibiotici ampiamente usati nelle infezioni causate, in modo documentato o sospetto, da patogeni Gram-positivi meticillino-resistenti. Tuttavia, l'emergere di ceppi di stafilococchi resistenti alla meticillina e a molti altri antibiotici con ridotta sensibilità ai glicopeptidi rappresenta una situazione critica che impone l'adozione di adeguate misure di vigilanza e controllo sulle infezioni nosocomiali e anche sulle infezioni domiciliari.

Sono molto pochi infatti gli antibiotici nuovi, disponibili già oggi o che lo saranno nei prossimi anni, attivi verso i patogeni Grampositivi meticillino-resistenti o multi-resistenti. Due soli antibiotici attivi nei confronti dei patogeni Gram-positivi resistenti sono stati introdotti in terapia negli ultimi anni: il quinupristin-dalfopristin e il linezolid [9-11].

Il linezolid, in particolare, è l'antibiotico capostipite di una nuova classe di farmaci, gli oxazolidinoni, la sola classe innovativa di antibiotici proposta negli ultimi trent' anni [12-15]. Gli oxazolidinoni, prodotti chimici di sintesi sviluppati inizialmente come inibitori delle
*Farmacologia Clinica Università di Torino 
monoamino-ossidasi per la terapia della depressione, hanno successivamente evidenziato una interessante attività antimicrobica, antibatterica e antifungina, ed alcuni di loro sono stati impiegati in agricoltura [12]. Dopo un lungo periodo di sviluppo è stato possibile identificare alcune molecole della classe dotate di elevata attività antibatterica, bassa tossicità e buone caratteristiche farmacocinetiche, ed una di queste, il linezolid, è stata sviluppata come antibiotico per uso umano [13].

Linezolid è dotato di un ampio spettro di attività nei confronti dei patogeni Gram-positivi, comprendente i ceppi di Staphylococcus aureus e di Staphylococcus epidermidis meticillino-resistente, i ceppi di Streptococcus pneumoniae penicillino- o eritromicina-resistenti, i ceppi di Enterococcus faecalis o Enteroccus faecium vancomicina-resistente [14-17].

Gli studi clinici di efficacia hanno dimostrato che linezolid è terapeuticamente equivalente agli antibiotici considerati standard di riferimento nella cura delle polmoniti e delle infezioni della cute e dei tessuti molli sostenute da Gram-positivi resistenti alla penicillina (ceftriaxone, cefpodoxime, oxacillina, dicloxacillina e claritromicina) o sostenute da MRSA (vancomicina) [15-19].

Un recente lavoro ha confrontato linezolid con vancomicina nel trattamento della pol- monite nosocomiale, evidenziando che nei pazienti con polmonite MRSA, il linezolid risulta superiore alla vancomicina in termini di percentuale di sopravvivenza e di successi clinici [20].

In uno studio successivo condotto dagli stessi autori, linezolid è risultato significativamente superiore alla vancomicina anche nei pazienti affetti da polmonite da ventilazione meccanica (VAP) dovuta a MRSA [21]. Inoltre, un recente studio multicentrico internazionale ha dimostrato che linezolid, a parità di sicurezza, è significativamente superiore, come efficacia, alla teicoplanina nel trattamento delle infezioni gravi sostenute da batteri Grampositivi, in particolare nei casi di batteriemia [22].

Linezolid è totalmente assorbito dal tratto gastrointestinale, perciò può essere somministrato anche per via orale, oltre che per via parenterale. La disponibilità di formulazioni sia parenterali sia orali consente di attuare terapie standard, solo endovenose o solo orali, oppure terapie sequenziali parenterali/orali senza dover cambiare antibiotico.

In questa rassegna presentiamo un breve profilo farmacoterapeutico e farmacoeconomico del linezolid, illustrandone soprattutto le caratteristiche peculiari che incidono sulla formazione dei costi sanitari a carico dell'ospedale e del SSN.

\begin{tabular}{lc}
\hline Patogeno & MIC-90 (mcg/ml) \\
\hline $\begin{array}{l}\text { Staphylococcus aureus inclusi i ceppi resistenti a meticillina, oxacillina o clindamicina e i ceppi con resistenza } \\
\text { intermedia a vancomicina }\end{array}$ & $\leq 4$ \\
Staphylococcus epidermidis e altri stafilococchi coagulasi-negativi, inclusi i ceppi resistenti a meticillina & $\leq 2$ \\
Enterococcus faecalis inclusi i ceppi resistenti all'ampicillina o alla vancomicina & $\leq 2$ \\
Enterococcus faecium inclusi i ceppi resistenti all'ampicillina o alla vancomicina & $\leq 2$ \\
Streptococcus pneumoniae (pneumococcus) resistente alla penicillina o multiresistente (a ceftriaxone, \\
claritromicina o ciprofloxacina) & $\leq 2$ \\
Streptococcus pyogenes ed altri streptococchi b-emolitici & $\leq 2$ \\
Bacillus spp. & $\leq 4$ \\
Corynebacterium spp. & $\leq 4$ \\
Lysteria monocytogenes & $\leq 4$ \\
Enterococcus raffinosus & $\leq 4$ \\
Pasteurella spp. inclusi i ceppi resistenti alla clindamicina & $\leq 4$ \\
Mycobacterium avium complex (MAC) & $\leq 4$ \\
\hline
\end{tabular}

Tabella 1

MIC-90 di linezolid nei confronti dei principali patogeni considerati sensibili [14, 15, 23-36] 


\section{PROFILO FARMACOLOGICO E TERAPEUTICO}

\section{Microbiologia}

Linezolid è un oxazolidinone dotato di elevata attività antibiotica verso la maggior parte dei batteri Gram-positivi, compresi quelli resistenti ad altri antibiotici, ma naturalmente resistente, in linea generale, alle enterobatteriacee e agli altri bacilli Gram-negativi [23-36]. La maggior parte dei cocchi Gram-positivi, compresi quelli resistenti ad altri antibiotici, viene inibita da concentrazioni di linezolid uguali o inferiori a $4 \mathrm{mcg} / \mathrm{ml}$. La tabella 1 riporta le concentrazioni minime inibenti del linezolid verso il $90 \%$ dei ceppi (MIC-90) dei patogeni considerati sensibili a questo antibiotico.

\section{Meccanismo d'azione e resistenze}

Gli oxazolidinoni sono inibitori della sintesi proteica a livello dei ribosomi batterici, ma si differenziano da tutti gli altri antibiotici che hanno come target i ribosomi perché bloccano la prima tappa del processo che porta all'assemblaggio dei ribosomi a partire dalle subunità dissociate. Il linezolid contrae un legame selettivo con l'RNA ribosomiale $23 \mathrm{~S}$ della subunità $50 \mathrm{~S}$ in un sito vicino all'interfaccia di questa con la subunità $30 \mathrm{~S}$, impedendo così la formazione del complesso 70S: ne consegue un blocco del processo di traslazione ribosomiale e della sintesi proteica [37-39].

Nessun altro antibiotico, tra quelli oggi noti, presenta questo stesso meccanismo e pertanto non vi sono resistenze crociate. Il cloramfenicolo e la lincomicina agiscono mediante un sito di legame vicino a quello del linezolid, situato nella parte centrale del dominio V dell'RNA ribosomiale $23 \mathrm{~S}$ della subunità $50 \mathrm{~S}$, dominio deputato alla formazione del legame peptidico. Il linezolid compete con il cloramfenicolo e la lincomicina su questo legame senza, tuttavia, inibire la formazione del legame peptidico e, soprattutto, senza indurre resistenze crociate con questi due antibiotici $[37,38]$.

Allo stato attuale delle conoscenze, linezolid sembra avere una minore tendenza a sviluppare resistenze. È difficile indurre resistenze batteriche al linezolid mediante passaggi seriali in vitro. Alcuni ceppi di patogeni resistenti al linezolid sono stati isolati dopo la sua commercializzazione. In particolare sono stati segnalati alcuni ceppi di Enterococcus faecium isolati da pazienti trattati per 21-40 giorni con linezolid perché il microrganismo era risultato resistente alla vancomicina [40]. Altri ceppi di enterococchi resistenti alla vancomicina (VRE) e al linezolid sono stati isolati da pazienti trapiantati [41].
Ceppi di MRSA resistenti al linezolid sono stati isolati dal liquido di perfusione di pazienti in dialisi peritoneale [42]. Le resistenze al linezolid sono state attribuite a mutazioni del DNA batterico codificanti il loop centrale del dominio $\mathrm{V}$ della frazione ribosomiale $23 \mathrm{~S}$. Le MIC in questi ceppi resistenti al linezolid erano superiori a $16 \mathrm{mcg} / \mathrm{ml}$.

\section{Farmacodinamica}

L'azione in vitro del linezolid è prevalentemente di tipo batteriostatico, anche se nei confronti degli streptococchi e dello $S$. aureus appare come lentamente battericida [35]. L'eradicazione di ceppi sensibili al linezolid avviene in tempi relativamente rapidi.

In uno studio clinico condotto su volontari con colonizzazione nasale di Staphylococcus aureus, la somministrazione di linezolid alle dosi di 200, 400 o 600mg ogni 12 ore per 3-5 giorni ha indotto la eradicazione nel $94 \%$ dei casi in un tempo mediano variabile da 36 a 60 ore [43].

In un ampio studio condotto su 288 pazienti valutabili, affetti da gravi infezioni e trattati in modo compassionevole con linezolid $600 \mathrm{mg}$ ogni 12 ore per via endovenosa o orale, è stata analizzata la correlazione tra farmacocinetica-farmacodinamica ed efficacia clinica. L'efficacia clinica è risultata correlata sia al rapporto AUC/MIC sia alla percentuale di tempo sopra-MIC. Nelle polmoniti, infezioni della cute e dei tessuti molli e batteriemie, si può avere una maggiore probabilità di successo clinico quando il rapporto AUC/MIC è almeno $80-120$ o quando i livelli plasmatici del linezolid permangono superiori alla MIC dei patogeni per l'intero intervallo interdose [44].

\section{Farmacocinetica}

Linezolid è assorbito rapidamente e quasi completamente dal tratto gastrointestinale [4546]. Dopo somministrazione di singola dose orale di 400 o $600 \mathrm{mg}$ si ottengono concentrazioni plasmatiche di picco superiori a $11-12 \mathrm{mcg} /$ $\mathrm{ml}$ entro 1-1,5 ore. Con dosi ripetute ogni 12 ore le concentrazioni raggiunte possono superare i $20 \mathrm{mcg} / \mathrm{ml}$ [46]. Somministrando una singola dose di $600 \mathrm{mg}$ di linezolid endovena in 30 minuti, le concentrazioni plasmatiche di antibiotico al termine dell'infusione raggiungono valori di circa $13 \mathrm{mcg} / \mathrm{ml}$ nell' adulto. Dopo dosi ripetute le concentrazioni plasmatiche al temine dell' infusione sono di circa $15 \mathrm{mcg} / \mathrm{ml}$.

Pertanto, una dose di linezolid somministrata per via orale induce mediamente curve di concentrazioni plasmatiche sovrapponibili a quelle ottenibili con la somministrazione della stessa dose per via endovenosa lenta [14]. 
Il cibo non interferisce significativamente con l'assorbimento e la biodisponibilità orale di linezolid. In presenza di cibi con elevati contenuti lipidici l'assorbimento risulta modicamente ritardato e le concentrazioni di picco ridotte, ma la quantità totale assorbita non varia. Linezolid ha un basso legame con le proteine plasmatiche (circa 31\%) e si distribuisce rapidamente ai tessuti ad alta perfusione. Il volume apparente di distribuzione allo steadystate nel paziente adulto è stato stimato in 4060 litri e nel bambino di età variabile da 3 mesi a 16 anni in $0,66 \mathrm{~L} / \mathrm{kg}$ [47-49].

Linezolid diffonde in modo adeguato anche in distretti profondi separati da barriere biologiche. Dopo 4 dosi da 600mg di linezolid somministrato ogni 12 ore per via endovenosa, nel liquido cerebrospinale sono state misurate concentrazioni di picco e di valle rispettivamente di 3,19 e 2,39 mcg/ml [50]. Nel liquido pleurico dosi terapeutiche di linezolid inducono concentrazioni uguali o superiori a $4 \mathrm{mcg} /$ $\mathrm{ml}$ [51]. Le concentrazioni di linezolid nel liquido di drenaggio del pancreas erano di 11-31 $\mathrm{mcg} / \mathrm{ml}$ dopo 3-7 giorni di trattamento alla dose di $600 \mathrm{mg}$ ogni 12 ore [52]. Linezolid è metabolizzato dal fegato in una quota oscillante dal $50 \%$ al $70 \%$ [53]. Il processo è saturabile alle dosi terapeutiche più elevate, ma ciò non implica alcuna conseguenza importante a livello clinico [48].

Attraverso processi ossidativi, che non coinvolgono il sistema dei citocromi P450, si formano diversi metaboliti, alcuni dei quali debolmente attivi. Non si ritiene, tuttavia, che i metaboliti attivi contribuiscano significativamente all' attività terapeutica del linezolid [53, 54]. L'escrezione di linezolid avviene per via renale in una quota di circa 1'80-85\% [53]. Circa il $30 \%$ della dose orale di linezolid viene escreto immodificato per via renale, mentre il $40 \%$ si ritrova nelle urine sotto forma del metabolica idrossietilglicina (PNU-142586) e il 10\% sotto forma del metabolica acido aminoetossiacetico (PNU-142300) [47, 53].

Una quota della dose orale variabile dal $6 \%$ al $12 \%$ viene escreta nelle feci, prevalentemente come metaboliti [53].
Il linezolid e i suoi metaboliti principali sono dializzabili. In tre ore di emodialisi viene rimosso oltre il $30 \%$ della dose: è stato stimato un rapporto di estrazione dialitica del $38 \%$, costante per tutta la seduta di dialisi. La clearance sistemica totale del linezolid è stata stimata in $80-146 \mathrm{ml} / \mathrm{min}$ nell' adulto e in $0,6 / \mathrm{L} / \mathrm{kg} / \mathrm{hr}$ nel bambino $[47,55]$. Un certo grado di nonlinearità della cinetica si manifesta a dosi elevate di linezolid, allorquando la clearance sistemica tende a diminuire. Questo risultato non ha comunque conseguenze pratiche a livello clinico [47, 48]. Inoltre la clearance sistemica del linezolid non risente in modo significativo della variazione di funzionalità renale e non varia sostanzialmente al variare della clearance della creatinina [55].

Analogamente si ritiene che la clearance sistemica del linezolid non sia significativamente influenzata da uno stato di insufficienza epatica, soprattutto se di entità lieve o moderata. Pertanto non è necessario modificare la posologia nei pazienti nefropatici o epatopatici, salvo nei casi estremi di insufficienza epatica per i quali vale il principio di prudenza.

L'emivita di eliminazione è mediamente di circa 5 ore nell' adulto e di poco meno di 3 ore nel bambino [47, 49]. Elevata variabilità interindividuale nell'emivita e nella clearance del linezolid è stata osservata in diversi gruppi di pazienti, compresi quelli affetti da fibrosi cistica [56,57].

In base all'emivita si può stimare che la condizione di accumulo si stabilizzi entro 2-4 giorni di trattamento, come dimostrano le curve di concentrazione ottenute dopo somministrazioni ripetute ogni 12 ore sia in soggetti sani sia nei diversi tipi di pazienti [46, 57]. L'emivita del linezolid è compatibile con lo schema posologico proposto, vhe prevede la somministrazione di una dose di linezolid ogni 12 ore: con la somministrazione, endovenosa o orale, di 400-600 mg ogni 12 ore l'accumulo è ben controllato e le concentrazioni plasmatiche, nell'intervallo tra le dosi, rimangono sopra i valori della MIC-90 dei patogeni sensibili per la maggior parte del tempo (tabella 2).

\begin{tabular}{lllll}
\hline Posologie & Concentrazioni plasmatiche & AUC $(\mathbf{m c g} / \mathbf{m l} h)$ & Emivita $(\mathrm{h})$ \\
\hline & Cmax $(\mathbf{m c g} / \mathbf{m l})$ & Cmin $(\mathbf{m c g} / \mathbf{m l})$ & & \\
600mg os ogni 12 ore & $21,20 \pm 5,78$ & $6,15 \pm 2,94$ & $138,00 \pm 42,10$ & $5,40 \pm 2,06$ \\
600 mg iv ogni 12 ore & $15,10 \pm 2,52$ & $3,68 \pm 2,36$ & $89,70 \pm 31,00$ & $4,80 \pm 1,70$ \\
\hline
\end{tabular}

Tabella 2

Parametri farmacocinetici di linezolid nell'adulto dopo dosi ripetute [11] 


\section{Efficacia terapeutica}

Le specialità medicinali a base di linezolid attualmente registrate in Italia hanno come indicazione le polmoniti nosocomiali e comunitarie e le infezioni della cute e dei tessuti molli [14]. In Italia l'uso di linezolid in pediatria non è ancora raccomandato, mentre negli Stati Uniti è consentito anche nel neonato [51]. La posologia raccomandata nell'adulto per trattare le polmoniti nosocomiali e comunitarie e le infezioni della cute e dei tessuti molli è di $600 \mathrm{mg}$ ogni 12 ore per via endovenosa o per via orale. Il trattamento antibiotico dovrebbe durare normalmente dai 10 ai 14 giorni, ma non dovrebbe superare i 28 giorni [14]. Queste indicazioni, contenute nella scheda tecnica di registrazione, derivano dall' analisi dei trials clinici finora effettuati per documentare l'efficacia e la tollerabilità del linezolid.

La tabella 3 riassume i risultati ottenuti con gli studi clinici comparativi di Fase III presentati per ottenere la registrazione del prodotto. I risultati di questi studi sono stati pubblicati anche su riviste internazionali di riferimento [20,21,58-66]. In questi studi registrativi di Fase III linezolid risulta equivalente o superiore, come efficacia, ai farmaci di confronto testati. Nel caso delle polmoniti comunitarie gli antibiotici di confronto sono stati due cefalosporine, ceftriaxone e cefpodoxime, che notoriamente non hanno alcuna attività nei confronti dei patogeni "atipici", clamidie, micoplasmi e legionelle. Inoltre, il linezolid non è molto attivo nei confronti dell' Haemophilus influenzae. Per questi motivi alcuni autori ri- tengono che il linezolid non possa essere considerato antibiotico di prima linea nelle polmoniti comunitarie [11].

D'altra parte il valore specifico di questo nuovo antibatterico consiste nella sua eccellente attività nei confronti di patogeni Grampositivi, come gli stafilococchi meticillino-resistenti o gli pneumococchi penicillino-resistenti, considerati "difficili" perché resistenti ai comuni antibiotici, tanto da richiedere l'impiego di antibiotici considerati di seconda linea, come i glicopeptidi vancomicina o teicoplanina, o come le cefalosporine di terza o quarta generazione usate per lo più in associazioni con altri antimicrobici. In effetti una delle indicazioni principali del linezolid è rappresentata dalla polmonite nosocomiale da Staphylococcus aureus meticillino-resistente, in alternativa alla vancomicina o alla teicoplanina.

Un recente lavoro ha analizzato retrospettivamente $i$ dati ottenuti in due studi prospettici internazionali, attuati a scopo registrativo, costruiti su un identico disegno a gruppi paralleli randomizzati in doppio cieco, e finalizzati a confrontare l'efficacia di linezolid con quella di vancomicina nel trattamento della polmonite nosocomiale [20]. La casistica complessiva esaminata includeva 1.019 pazienti con sospetta polmonite nosocomiale, randomizzati nei due gruppi, trattati per via endovenosa rispettivamente con linezolid $600 \mathrm{mg}(\mathrm{n}=524)$ o con vancomicina $1 \mathrm{~g}(\mathrm{n}=495)$, due volte al giorno per un periodo compreso tra 7 e 21 giorni. L'aztreonam, 1-2 g ogni 8 ore, è stato associato

\begin{tabular}{|c|c|c|c|c|c|}
\hline Studio & Antibiotici & $\begin{array}{l}\text { Posologie } \\
\text { giornaliere }\end{array}$ & $\begin{array}{l}\text { Giorni } \\
\text { terapia }\end{array}$ & Successi $n / n(\%)$ & $\mathbf{P}$ \\
\hline \multirow[t]{2}{*}{ Polmoniti nosocomiali } & Linezolid* & $600 \mathrm{mg} \times 2$ & $7-21$ & $221 / 417(53,0 \%)$ & \multirow{2}{*}{ NS } \\
\hline & Vancomicina* & $\lg \times 2$ & $7-21$ & $202 / 387(52,2 \%)$ & \\
\hline \multirow[t]{2}{*}{ Polmoniti comunitarie ospedalizzate } & Linezolid $^{\circ}$ & $600 \mathrm{mg} \times 2$ & $7-14$ & $247 / 272(90,8 \%)$ & \multirow[b]{2}{*}{ NS } \\
\hline & $\begin{array}{l}\text { Ceftriaxone iv/ } \\
\text { Cefpodoxime os }\end{array}$ & $\begin{array}{l}1 \mathrm{~g} \times 2 / \\
200 \mathrm{mg} \times 2\end{array}$ & $7-14$ & $225 / 254(88,6 \%)$ & \\
\hline Polmoniti comunitarie a domicilio & $\begin{array}{l}\text { Linezolid } \\
\text { Cefpodoxime os }\end{array}$ & $\begin{array}{l}600 \mathrm{mg} \times 2 \\
200 \mathrm{mg} \times 2\end{array}$ & $\begin{array}{l}10-14 \\
10-14\end{array}$ & $\begin{array}{l}180 / 201(89,6 \%) \\
187 / 206(90,8 \%)\end{array}$ & NS \\
\hline \multirow[t]{2}{*}{ Infezioni complicate della cute e tessuti molli } & Linezolid $^{\circ}$ & $600 \mathrm{mg} \times 2$ & $10-21$ & $264 / 291(90,7 \%)$ & \multirow[b]{2}{*}{ NS } \\
\hline & $\begin{array}{l}\text { Oxacillina iv/ } \\
\text { Dicloxacillina os }\end{array}$ & $\begin{array}{l}2 \mathrm{~g} / \\
500 \mathrm{mg}\end{array}$ & $10-21$ & $259 / 300(86,3 \%)$ & \\
\hline Infezioni non complicate della cute e tessuti molli & $\begin{array}{l}\text { Linezolid } \\
\text { Claritromicina os }\end{array}$ & $\begin{array}{l}400 \mathrm{mg} \times 2 \\
250 \mathrm{mg} \times 2\end{array}$ & $\begin{array}{l}7-14 \\
7-14\end{array}$ & $\begin{array}{l}183 / 310(91,3 \%) \\
262 / 301(87,0 \%)\end{array}$ & 0,09 \\
\hline
\end{tabular}

* In associazione con aztreonam

${ }^{\circ}$ Con eventuale associazione di aztreonam in caso di bacilli gram-negativi

\section{Tabella 3}

Studi clinici comparativi di Fase III effettuati per documentare l'efficacia terapeutica di linezolid nelle polmoniti nosocomiali, comunitarie e nelle infezioni della cute e dei tessuti molli (modificata da: Moellering RC et al, 2003) [11] 


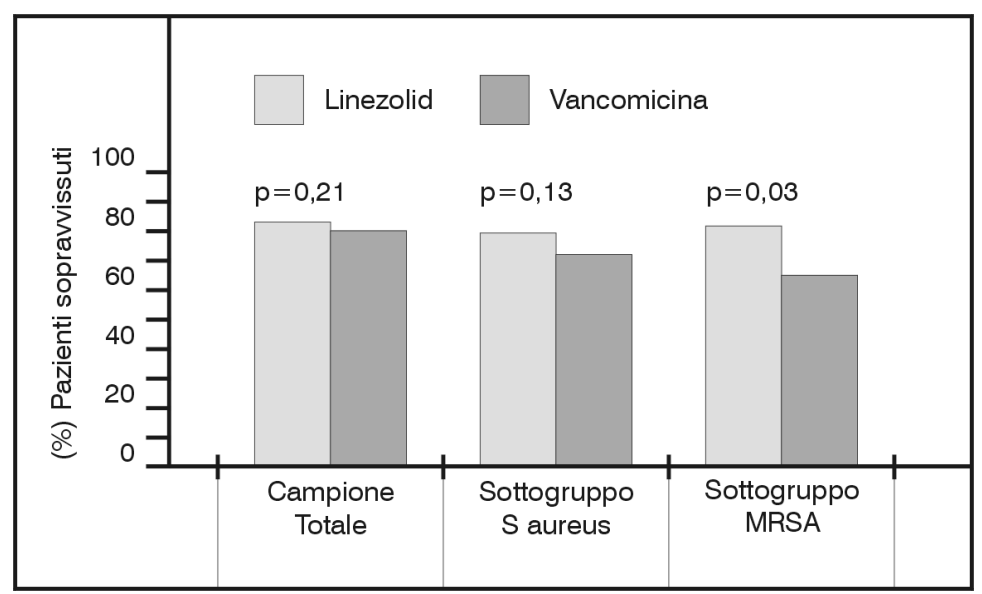

Figura 1

Percentuali di sopravvivenza nei pazienti affetti da polmonite nosocomiale, trattati per via endovenosa con linezolid o con vancomicina, in associazione con aztreonam: risultati dell'analisi retrospettiva condotta secondo ITT sul campione totale e su sottogruppi di pazienti inclusi in due identici studi clinici multicentrici internazionali di Fase III con disegno in doppio cieco a gruppi paralleli randomizzati (elaborata da: Wunderink RG et al, 2003) [20]

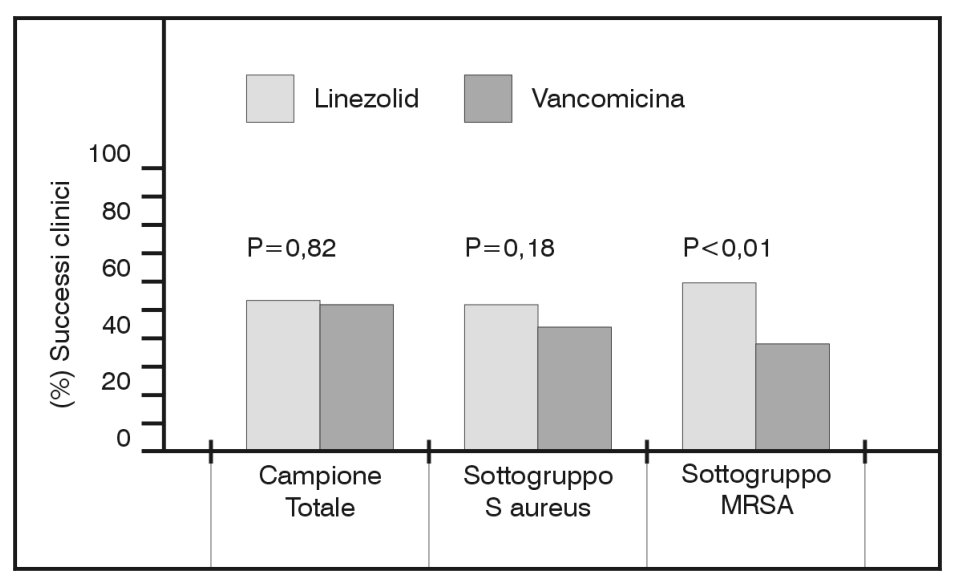

\section{Figura 2}

Percentuali di successi clinici nei pazienti affetti da polmonite nosocomiale, trattati per via endovenosa con linezolid o con vancomicina, in associazione con aztreonam: risultati dell'analisi retrospettiva condotta secondo ITT sul campione totale e su sottogruppi di pazienti inclusi in due identici studi clinici multicentrici internazionali di Fase III con disegno in doppio cieco a gruppi paralleli randomizzati (elaborata da: Wunderink RG et al, 2003) [20]

in tutti i pazienti e sospeso solo dopo aver stabilito che non vi fossero Gram-negativi tra i possibili co-patogeni. La presenza di S. aureus è stata documentata in 366 pazienti (sottogruppo S. aureus) e la polmonite da MRSA in 160 (sottogruppo MRSA). Lo studio ha valutato, secondo ITT, le percentuali di sopravvivenza nell'intero campione e nei due sottogruppi, nonché le percentuali di successi clinici (cura della polmonite), dopo l'esclusione dei pazienti non valutabili ai fini dell'efficacia. I risultati principali sono riassunti nelle figure 1 e 2.

Considerando il campione totale o il sottogruppo $S$. aureus, i tassi di sopravvivenza e di cura dopo linezolid e dopo vancomicina sono equivalenti. Tuttavia, quando si considera il sottogruppo di polmoniti sostenute da MRSA, il linezolid risulta significativamente superiore alla vancomicina sia in relazione alla percentuale di sopravvivenza sia in relazione alla percentuale di successi clinici [20]. In un successivo studio, appena pubblicato, gli stessi autori hanno confermato questi risultati sul sottocampione di pazienti affetti da VAP: in confronto con vancomicina, la terapia iniziale con linezolid è associata a tassi significativamente superiori di sopravvivenza e di cura nel sottogruppo di pazienti affetti da VAP dovuta a MRSA [21]. In entrambi i lavori, inoltre, linezolid risulta tendenzialmente più efficace della vancomicina, senza raggiungere la significatività statistica, anche quando si considera l'intero campione o il sottocampione $S$. aureus.

L'analisi della regressione logistica ha dimostrato che il linezolid è un predittore indipendente della probabilità di sopravvivenza e di cura con valori di odds ratio crescenti passando dall'intero campione al sottogruppo $S$. aureus, quindi al sottogruppo MRSA [20,21]. Un importante studio clinico di Fase III, multicentrico, internazionale e randomizzato, ha confrontato l'efficacia clinica del linezolid (600mg iv/os ogni 12 ore) con quella della vancomicina (1g iv ogni 12 ore), nel trattamento ospedaliero delle infezioni dovute a stafilococchi meticillino-resistenti (MRSS), focalizzando principalmente l'analisi sui vantaggi della "switch-therapy" iv/os (possibile con linezolid senza dover cambiare antibiotico), e stimando la durata delle degenze e i giorni di terapia antibiotica con linezolid (per via endovenosa e orale) o vancomicina (per via endovenosa) [65]. Nello studio sono stati inclusi 460 pazienti (254 valutabili clinicamente), di età >13 anni, affetti da infezione stafilococcica, sospetta o documentata. La casistica comprendeva polmoniti $(22 \%)$, infezioni complicate della cute e tessuti molli $(50 \%$ circa), delle vie urinarie (6\%), batteriemie di origine sconosciuta $(11 \%)$ e varie altre infezioni. Ceppi MRSA sono stati isolati nel $49 \%$ dei pazienti, altri stafilococchi nel $17 \%$, mentre nel restante $34 \%$ dei pazienti non è stato possibile isolare alcun microrganismo.

La tabella 4 riporta i giorni di degenza e i giorni di terapia nei due gruppi, raggruppando il campione secondo vari criteri: campione totale e stratificato per sede d'infezione, includendo i soggetti valutabili secondo ITT o per efficacia clinica. Considerando l'intero campione secondo ITT, la durata mediana della degenza, sebbene tendenzialmente inferiore nel gruppo trattato con linezolid, non è risultata significativamente differente da quella del gruppo trattato con vancomicina. Al contra- 
rio, nelle infezioni complicate della cute e dei tessuti molli la durata mediana delle degenze nel gruppo linezolid è risultata inferiore di 5 giorni ( $\mathrm{p}=0,05)$, quando si considerava il campione secondo ITT, o di 8 giorni ( $\mathrm{p}=0,0025)$, quando si considerava il sottocampione valutabile clinicamente (tabella 4 ).

Durante la prima settimana la percentuale di pazienti dimessi dall'ospedale è risultata significativamente superiore nel gruppo trattato con linezolid, sia includendo i pazienti valutabili secondo ITT (30\% vs 19\%, p=0,005) (figura 3), sia includendo solo quelli valutabili clinicamente $(31 \%$ vs $12 \%, p=0,005)$. Nel sottocampione dei pazienti affetti da infezioni della cute e tessuti molli le percentuali di dimissioni del gruppo linezolid permanevano significativamente superiori anche nella seconda settimana di trattamento, quando si includevano i pazienti valutabili clinicamente (figura 4).

La durata media o mediana della terapia antibiotica per via endovenosa è risultata significativamente inferiore nel gruppo trattato

\begin{tabular}{|c|c|c|c|c|c|c|}
\hline & \multicolumn{3}{|c|}{ Intention-to-Treat } & \multicolumn{3}{|c|}{ Valutabili clinicamente } \\
\hline & LIN & VAN & $\mathbf{P}$ & LIN & VAN & $\mathbf{P}$ \\
\hline \multicolumn{7}{|l|}{ Tutte le infezioni } \\
\hline$N^{\circ}$ pazienti & 240 & 220 & - & 124 & 130 & - \\
\hline \multicolumn{7}{|l|}{ Giorni degenza } \\
\hline Media & 19,6 & 20,2 & & 20,0 & 21,3 & \\
\hline Mediana $(95 \% \mathrm{Cl})$ & $14(9-17)$ & $15(13-17)$ & 0,19 & $14(9-18)$ & $16(14-19)$ & 0,08 \\
\hline \multicolumn{7}{|l|}{ Giorni terapia antibiotica } \\
\hline \multicolumn{7}{|l|}{ Endovenosa } \\
\hline Media & 6,7 & 11,3 & 0,0001 & 6,8 & 13,8 & 0,0001 \\
\hline Mediana & 5 & 10 & 0,0001 & 6 & 13 & 0,0001 \\
\hline \multicolumn{7}{|c|}{ Totale endovenosa + orale } \\
\hline Media & 12,6 & 11,3 & 0,05 & 15,4 & 13,8 & 0,02 \\
\hline Mediana & 13 & 10 & 0,03 & 15 & 13 & 0,003 \\
\hline \multicolumn{7}{|c|}{ Infezioni complicate della cute e tessuti molli } \\
\hline$N^{0}$ pazienti & 122 & 108 & - & 70 & 74 & - \\
\hline \multicolumn{7}{|l|}{ Giorni degenza } \\
\hline Media & 17,2 & 19,4 & & 15,4 & 20,3 & \\
\hline Mediana $(95 \% \mathrm{Cl})$ & $9(8-16)$ & $14(11-18)$ & 0,052 & $8(6-13)$ & $16(13-19)$ & 0,0025 \\
\hline \multicolumn{7}{|l|}{ Giorni terapia antibiotica } \\
\hline \multicolumn{7}{|l|}{ Endovenosa } \\
\hline Media & 5,8 & 12,6 & 0,0001 & 5,4 & 14,8 & 0,0001 \\
\hline Mediana & 4 & 11 & 0,0001 & 4 & 14 & 0,0001 \\
\hline \multicolumn{7}{|c|}{ Totale endovenosa + orale } \\
\hline Media & 14,2 & 12,6 & 0,09 & 16,6 & 14,8 & 0,07 \\
\hline Mediana & 14 & 11 & 0,056 & 15 & 14 & 0,027 \\
\hline \multicolumn{7}{|l|}{ Altre infezioni } \\
\hline$N^{\circ}$ pazienti & 118 & 112 & - & 54 & 56 & - \\
\hline Giorni degenza & 21,6 & 20,7 & & 25,7 & 22,0 & \\
\hline $\begin{array}{l}\text { Media } \\
\text { Mediana }(95 \% \text { Cl) }\end{array}$ & $16(12-21)$ & $15(12-21)$ & 0,99 & $18(14-29)$ & $15(13-25)$ & 0,46 \\
\hline \multicolumn{7}{|l|}{ Giorni terapia antibiotica } \\
\hline \multicolumn{7}{|l|}{ Endovenosa } \\
\hline Media & 7,6 & 10,1 & 0,0005 & 8,7 & 12,5 & 0,0001 \\
\hline Mediana & 7 & 10 & 0,0005 & 7 & 12 & 0,0001 \\
\hline \multicolumn{7}{|c|}{ Totale endovenosa + orale } \\
\hline Media & 10,9 & 10,1 & 0,33 & 13,8 & 12,5 & 0,13 \\
\hline Mediana & 10,5 & 10 & 0,28 & 14 & 12 & 0,054 \\
\hline
\end{tabular}

\section{Tabella 4}

Durata della degenza e durata della terapia antibiotica nei pazienti affetti da infezioni sostenute da MRSS, trattati con linezolid iv/os e nei pazienti trattati con vancomicina iv (modificata da: Li Z et al, 2001) [65] 


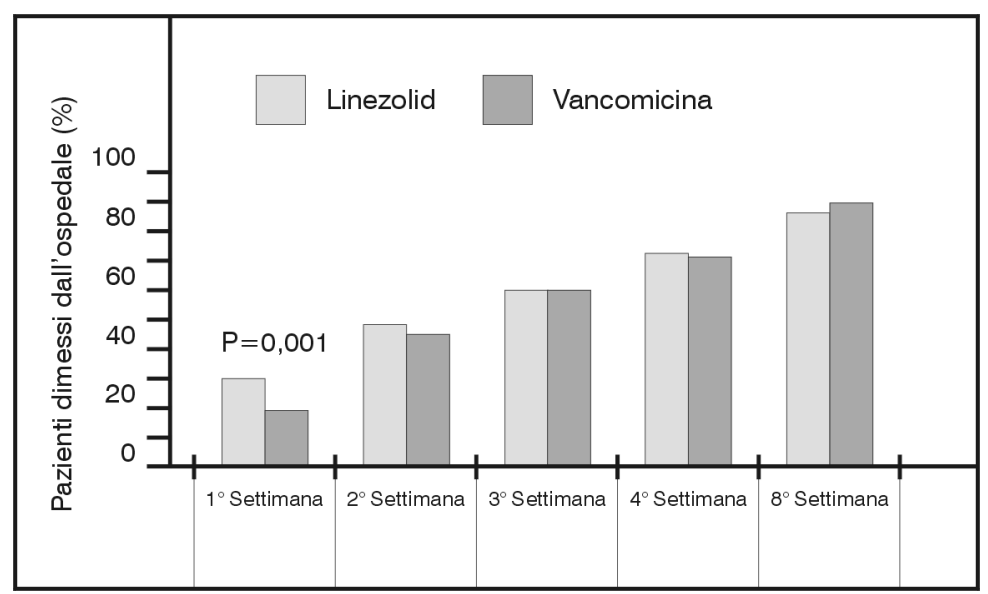

Figura 3

Percentuali cumulative di dimissioni nelle settimane dall'inizio della terapia con linezolid iv/os o con vancomicina iv: campione totale includendo i soggetti valutabili secondo ITT (dati da: Li Z et al, 2001) [65]

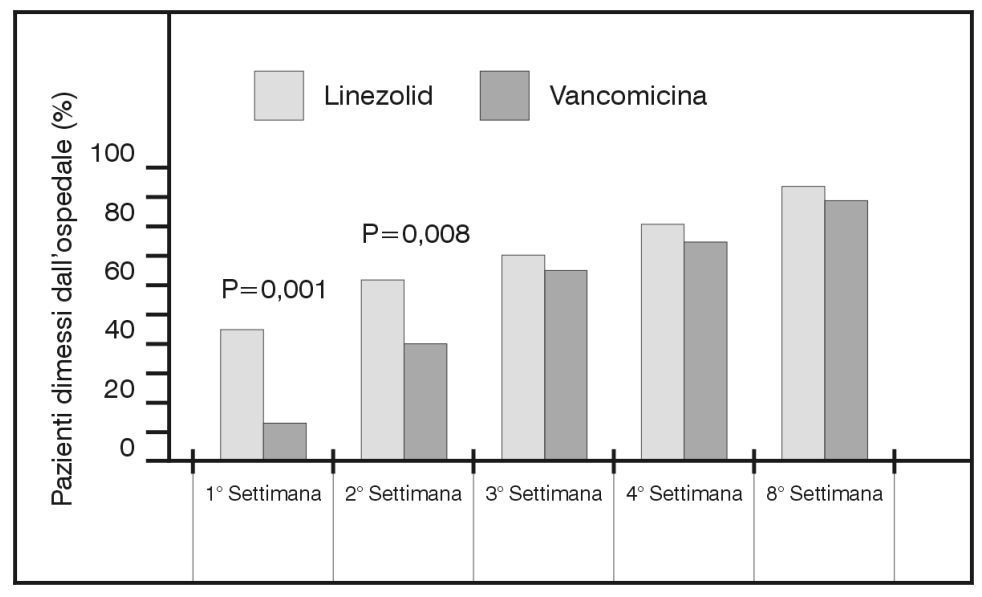

\section{Figura 4}

Percentuali cumulative di dimissioni dall'ospedale nelle settimane dopo l'inizio della terapia con linezolid iv/os o con vancomicina iv: campione dei pazienti affetti da infezioni complicate della cute e tessuti molli includendo i soggetti valutabili clinicamente (dati da: Li Z et al, 2001) [65]

con linezolid rispetto al gruppo trattato con vancomicina, indipendentemente dalla sede di infezione e dai criteri di valutazione del campione (tabella 4). Nel gruppo linezolid, il 60,8\% dei pazienti valutati secondo ITT e l' $81,4 \%$ dei pazienti valutabili clinicamente è stato trattato anche per via orale. La durata complessiva della terapia con linezolid (endovenosa + orale) è risultata significativamente superiore, mediamente di 1-2 giorni, rispetto alla durata complessiva della terapia con vancomicina.

Questi risultati sono stati confermati anche dopo aver escluso i casi di decessi.

La disponibilità di una formulazione orale di linezolid e la possibilità di adottare la "switchtherapy" iv/os senza dover cambiare antibiotico è uno dei fattori principali che condiziona la minor durata della degenza e i potenziali significativi risparmi per l'ospedale e per il SSN (vedi oltre).
L'efficacia terapeutica del linezolid è stata confrontata con quella della teicoplanina in uno studio multicentrico internazionale, controllato e randomizzato, condotto in aperto su un gruppo di 430 pazienti affetti da infezioni sostenute da sospetti o documentati patogeni Gram-positivi [66]. Il campione includeva pazienti affetti da polmoniti acquisite in comunità, da infezioni della cute e dei tessuti molli e da batteriemie. Metà dei pazienti sono stati trattati con linezolid $600 \mathrm{mg}$ ogni 12 ore per via endovenosa e/o orale e l'altra metà con teicoplanina per via endovenosa o intramuscolare (200-800mg/die), per un periodo variabile da 7 a 28 giorni. Circa il $90 \%$ dei pazienti di ciascun gruppo è stato trattato per almeno 7 giorni. La maggior parte dei pazienti $(82 \%)$ del gruppo linezolid è passato alla via orale dopo 6-8 giorni di terapia endovenosa.

L'efficacia clinica comparativa di linezolid e teicoplanina è riassunta nella figura 5 . Secondo ITT, alla visita di fine trattamento il linezolid è risultato significativamente più efficace della teicoplanina, quando si considera l'intero campione o il sottocampione di pazienti con batteriemia, mentre è risultato equivalente alla teicoplanina quando si considera il gruppo di pazienti affetti da polmonite o da infezioni della cute e dei tessuti molli (figura 5). Con l'uso di linezolid il tasso di eradicazione è risultato tendenzialmente, seppur non significativamente, superiore per tutti i patogeni e per tutte le sedi di infezione, fatta eccezione per lo $S$. pneumoniae e per le polmoniti [66].

Un recente studio clinico multicentrico, randomizzato in doppio cieco, ha misurato l'efficacia terapeutica di linezolid e di teicoplanina in un gruppo di 202 pazienti critici affetti da gravi infezioni sostenute da sospetti o documentati patogeni Gram-positivi. Il campione includeva pazienti affetti da polmonite nosocomiale, infezioni della cute e dei tessuti molli, batteriemie, osteomieliti o artriti settiche [67]. Tutti i pazienti sono stati trattati per via endovenosa, un gruppo di 100 con linezolid alla dose standard di $600 \mathrm{mg}$ ogni 12 ore e un gruppo di 102 con teicoplanina alla dose di $400 \mathrm{mg}$ ogni 12 ore per tre giorni e di $400 \mathrm{mg} / \mathrm{die}$ nei giorni successivi. Sono stati associati altri antibiotici per ampliare la copertura dello spettro, trattandosi di terapie empiriche. In questo studio linezolid ha dimostrato efficacia e tollerabilità equivalenti a quelle della teicoplanina. Al termine del trattamento i successi clinici ammontavano al 78,9\% nel gruppo linezolid e al 72,8\% nel gruppo teicoplanina, mentre i successi microbiologici erano rispettivamente del $70 \%$ e del $66,2 \%$. Analogamente anche i successi valutati a breve e lungo termine, le mortalità in unità di terapia intensiva e gli 
eventi avversi risultarono equivalenti. L'unica differenza statisticamente significativa tra linezolid e teicoplanina risultò essere il tasso di riduzione della colonizzazione da MRSA entro il periodo di trattamento, superiore con linezolid $(51,1 \%$ vs $18,6 \%, p=0,002)$ [67].

\section{Tollerabilità e sicurezza}

I dati finora disponibili indicano che il profilo di tollerabilità di sicurezza clinica del linezolid è equivalente a quello dei farmaci con i quali è stato confrontato. Un recente lavoro ha valutato questo tema utilizzando la casistica di oltre 4.000 pazienti raccolta nel corso di 7 studi clinici controllati di Fase III [68]. La casistica comprende pazienti affetti da polmoniti nosocomiali e comunitarie, infezioni della cute e dei tessuti molli e infezioni sostenute da MRSA. La casistica è anche eterogenea per quanto riguarda la gravità dei casi clinici. Il campione è costituito complessivamente da 4.047 pazienti: 2.046 trattati con linezolid e 2.001 con i farmaci di confronto, cioè vancomicina, ceftriaxone, cefpodoxime, oxacillinadicloxacillina e claritromicina. Nel pool dei pazienti trattati con linezolid il $19 \%$ ha ricevuto il farmaco solo per via endovenosa, il $40 \%$ solo per via orale e il rimanente $41 \%$ per via endovenosa seguita dalla via orale. La durata media di trattamento (11,6 giorni) è risultata sovrapponibile nel gruppo linezolid e nel gruppo di confronto.

I principali risultati di questa indagine sono riassunti in tabella 5 . Le frequenze di eventi avversi gravi $(11,4 \%$ vs $10,6 \%)$, di morti $(4,8 \%$ vs $4,9 \%)$ e di sospensione del farmaco per intolleranza al farmaco $(2,4 \%$ vs $1,9 \%)$ erano equivalenti nei due gruppi. Prescindendo dall'attribuzione al farmaco, gli eventi avversi più frequentemente segnalati nel gruppo linezolid e nel gruppo di confronto sono stati diarrea $(8,3 \%$ vs $6,3 \%, \mathrm{p}=0,018)$, cefalea ( $6,5 \%$ vs $5,5 \%, \mathrm{p}=0,155)$ e nausea $(6,2 \%$ vs $4,6 \%, \mathrm{p}=0,024)$. Diverse altre reazioni avverse si sono manifestate con frequenze comprese tra $0,1 \%$ e $1 \%$ dei pazienti, in entrambi i gruppi. Le reazioni avverse più comuni al linezolid sono indipendenti dalla via di somministrazione. Il farmaco, inoltre è ben tollerato, a livello locale e sistemico, quando somministrato per via endovenosa. In caso di somministrazione per via orale sono frequenti le classiche reazioni avverse, tipiche degli antibiotici orali; queste reazioni avverse sono responsabili della maggior frequenza complessiva di eventi avversi osservati nel gruppo linezolid rispetto al gruppo di confronto, tenuto conto che la percentuale di pazienti trattati con antibiotico orale nel gruppo di confronto è nettamente inferiore.

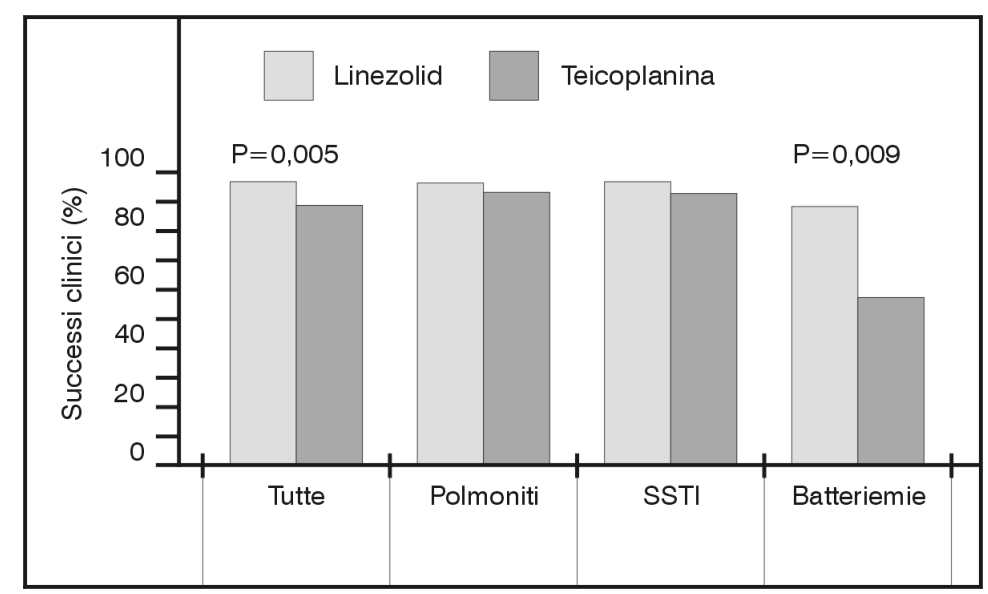

Figura 5

Percentuali di successi clinici, per sede di infezione, alla visita di fine trattamento con linezolid o con teicoplanina (da: Wilcox M et al, 2004) [66]

In compenso, nel gruppo linezolid è leggermente inferiore la frequenza di complicazioni correlate al Clostridium difficile.

Le alterazioni dei parametri chimico-clinici ed ematologici testati dal laboratorio non sono risultate significativamente differenti nel gruppo linezolid e nel gruppo di confronto. Inoltre sono state transitorie e raramente hanno comportato la sospensione del farmaco. Unica segnalazione degna di nota è una tendenza alla riduzione delle piastrine con l'uso prolungato di linezolid. Le caratteristiche delle anomalie ematologiche osservate, il tempo di comparsa, la reversibilità dopo sospensione del farmaco fanno ipotizzare che all'uso di linezolid sia associato un rischio di mielosoppressione reversibile, correlata alla durata del trattamento oltre i 14 giorni. Questa ipotesi è confermata da segnalazioni spontanee nel corso della farmacovigilanza post-marketing, da risultati ottenuti nel corso di programmi di uso compassionevole prolungato del farmaco e da report comparsi recentemente nella letteratura scientifica [69-74].

I casi di segnalazione spontanea di trombocitopenia e/o anemia associati a linezolid sono poco frequenti, sono sempre stati reversibili dopo sospensione del farmaco e sono stati osservati in pazienti trattati per oltre 14 giorni.

Pertanto, se la terapia con linezolid deve essere prolungata è raccomandato il monitoraggio ematologico periodico. Il rischio di mielosoppressione da linezolid è giudicato pienamente accettabile in relazione al potenziale beneficio di questo nuovo antibatterico nei confronti di patogeni Gram-positivi, inclusi microrganismi resistenti problematici come i MRSA, lo S. pneumoniae penicillinoresistente e gli enterococchi vancomicino-resistenti [68]. 


\section{Interazioni farmacologiche}

Linezolid conserva una scarsa attività di inibizione aspecifica e reversibile sulle monoamino-ossidasi (MAO). Vi è quindi un rischio, seppur ridotto, di interazione con farmaci adrenergici e serotoninergici [75, 76].

Somministrato da solo non ha dimostrato di avere effetti significativi sulla pressione arteriosa [76]. Tuttavia, l'associazione con farmaci adrenergici o serotoninergici e con cibi ad alto contenuto in tiramina può comportare incrementi significativi della pressione [14]. Linezolid deve esser usato con cautela nei pazienti in trattamento con un antidepressivo SSRI per il rischio, peraltro molto ridotto, di veder emergere una sindrome serotoninica (oltre ad ipertensione, anche iperpiressia, sudorazione, brividi, eccitazione, tremori, mioclonie) [77-79].

Il rischio di reazioni avverse da interazioni farmacologiche è giudicato comunque molto basso. Nell'ambito dell' analisi sul profilo di sicurezza, condotta sul pool di dati ottenuti in 7 studi di Fase III, è stato osservato che circa il $30 \%$ dei pazienti del gruppo linezolid e del gruppo di confronto avevano ricevuto associazioni con farmaci potenzialmente interferenti con inibitori delle MAO (simpaticomimetici, vasopressori, antidepressivi, stimolanti, antitosse, analgesici, ecc.). Reazioni avverse ai farmaci teoricamente correlabili alle MAO, come rialzi pressori, ipertermia e palpitazioni, sono stati osservati con frequenze $<1 \%$ in en- trambi i gruppi, senza differenze significative, sebbene gli antibiotici di confronto non abbiano alcuna attività inibitrice sulle MAO [68].

\section{PROFILO FARMACOECONOMICO}

L'analisi farmacoeconomica, inserendosi nella nobile ma complessa attività del processo decisionale, non può limitarsi alla sola valutazione dei costi, per lo più parziali, delle terapie farmacologiche, ma deve analizzare l'insieme dei fattori e dei valori che possono determinare la scelta tra alternative in campo sanitario. Una tecnica propedeutica alle tradizionali tecniche di analisi costo/efficacia, utile quando la decisione è complessa ed è opportuno evidenziare e pesare i fattori rilevanti per i diversi decisori che intervengono nelle scelte sanitarie, è rappresentata dalla "analisi dei valori” [80]. Questa tecnica consiste nel formulare e testare un insieme di valori proposti che variano in funzione del tipo di outcome clinico ed economico e in funzione delle diverse prospettive dei decisori coinvolti o attori interessati alla decisione.

Il presupposto è che un outcome positivo o negativo per un paziente potrebbe avere un significato molto differente per il medico, per l'ospedale, per il SSN o per altri decisori. Considerando di dover analizzare il valore, positivo e negativo, attribuito da ognuno dei decisori all'outcome sanitario e all'outcome economico di ciascuna alternativa, è utile costruire una matrice, come quella rappresentata

\begin{tabular}{|c|c|c|c|c|}
\hline \multirow[t]{2}{*}{ Reazione avversa al farmaco } & \multicolumn{2}{|c|}{ Pazienti trattati con linezolid $(n=2.046)$} & \multicolumn{2}{|c|}{ Altri antibiotici di confronto $(n=2.001)$} \\
\hline & $\begin{array}{l}\text { Con una o più } \\
\text { reazione avversa } \\
(n=444)\end{array}$ & $\begin{array}{l}\text { Sospensione } \\
\text { farmaco } \\
(n=50)\end{array}$ & $\begin{array}{l}\text { Con una o più } \\
\text { reazione avversa } \\
(n=314)\end{array}$ & $\begin{array}{l}\text { Sospensione } \\
\text { farmaco } \\
(n=38)\end{array}$ \\
\hline Diarrea & $4,3 \%$ & $0,3 \%$ & $3,2 \%$ & $0,2 \%$ \\
\hline Nausea & $3,4 \%$ & $0,5 \%$ & $2,3 \%$ & $0,1 \%$ \\
\hline Cefalea & $2,2 \%$ & $0,4 \%$ & $1,3 \%$ & $<0,1 \%$ \\
\hline Gusto amaro & $1,2 \%$ & $0,0 \%$ & $0,7 \%$ & $<0,1 \%$ \\
\hline Mobiliasi vaginale & $1,2 \%$ & $0,0 \%$ & $0,6 \%$ & $0,0 \%$ \\
\hline Vomito & $1,1 \%$ & $0,3 \%$ & $0,4 \%$ & $0,1 \%$ \\
\hline Funzione epatica alterata & $1,0 \%$ & $0,1 \%$ & $0,3 \%$ & $<0,1 \%$ \\
\hline
\end{tabular}

\section{Tabella 5}

Reazioni avverse al farmaco riportate dai ricercatori con frequenze $>1 \%$ nel pool di pazienti trattati con linezolid e nel pool di pazienti trattati con altri antibiotici di confronto e relative percentuali di sospensione del farmaco [68] 
in tabella 6. Per ogni decisore considerato, le quattro caselle contengono il giudizio di valore relativo all'incrocio tra tipo di outcome (clinico ed economico) e segno positivo o negativo dell'outcome stesso: questi giudizi possono essere ottenuti in vario modo, ad esempio analizzando esaustivamente la letteratura disponibile. Dall'analisi della matrice dei valori (tabella 6) che abbiamo costruito considerando il profilo terapeutico sopra riassunto, emerge chiaramente quali possono essere $\mathrm{i}$ vantaggi sanitari del linezolid rispetto ai farmaci coi quali compete e rispetto ai quali è stato confrontato (principalmente vancomicina e teicoplanina):

1. equivalente o superiore probabilità di sopravvivenza all'infezione, con guadagno di anni di vita;

2. efficacia clinica e microbiologica equivalente o superiore nel trattamento delle gravi infezioni sostenute da Gram-positivi resistenti;

3. efficacia clinica e microbiologica superiore nel trattamento delle polmoniti nosocomiali da MRSA;
4. possibilità di utilizzare la via orale e di attuare protocolli di "switch-therapy" iv/os;

5. possibilità di ridurre la durata della degenza e continuare la terapia a domicilio;

6. guadagno in qualità di vita.

Questi vantaggi rappresentano un valore sanitario ed etico rilevante ma, dato l'elevato costo del linezolid, il loro raggiungimento comporta verosimilmente un sensibile incremento dei costi diretti sanitari a carico dell'ospedale e del SSN.

Alcuni lavori pubblicati negli ultimi anni hanno esaminato la costo/efficacia del linezolid nel tentativo di valutare quando e quanto sia compatibile, per i bilanci degli ospedale e dei SSN, l'uso di linezolid nel trattamento delle polmoniti e delle infezioni sostenute da patogeni Gram-positivi resistenti o multiresistenti, in alternativa alla vancomicina e alla teicoplanina [81-85].

Di seguito tracciamo una breve rassegna critica di questi lavori.

\begin{tabular}{|c|c|c|}
\hline \multicolumn{3}{|c|}{ Prospettiva del paziente } \\
\hline Valore & Positivo & Negativo \\
\hline Clinico & $\begin{array}{l}\text { Probabilità equivalente o maggiore di guarire e di } \\
\text { soprawivere a gravi infezioni batteriche } \\
\text { Possibilità di essere trattato per via orale senza } \\
\text { riduzione di efficacia } \\
\text { Possibilità di essere dimesso prima dall'ospedale } \\
\text { Facilità di continuare a domicilio la cura e migliore } \\
\text { qualità di vita }\end{array}$ & $\begin{array}{l}\text { Necessità di associazioni quando la terapia non è mirata } \\
\text { ma empirica } \\
\text { Maggior frequenza di reazioni awverse non gravi da } \\
\text { assunzione orale } \\
\text { Rischio limitato e controllabile di trombocitopenia e } \\
\text { mielosoppressione reversibile } \\
\text { Rischio di interazioni con antidepressivi (SSRI) }\end{array}$ \\
\hline Economico & $\begin{array}{l}\text { Nessun costo diretto a carico } \\
\text { Possibile riduzione dei costi indiretti per una minore } \\
\text { durata dell'inabilità lavorativa }\end{array}$ & $\begin{array}{l}\text { Possibile aumento dei costi indiretti in caso di reazioni } \\
\text { awerse }\end{array}$ \\
\hline
\end{tabular}

\section{Tabella $6 a$}

Matrice dei valori per giudicare la convenienza ad usare linezolid nelle polmoniti nosocomiali e comunitarie, nelle infezioni della cute e dei tessuti molli, sostenute da patogeni Gram-positivi resistenti ai comuni antibiotici - Prospettiva del paziente

\begin{tabular}{lll}
\hline Prospettiva del medico curante (ospedaliero e territoriale) & \\
\hline Valore & Positivo & Negativo \\
Clinico & $\begin{array}{l}\text { Probabilità equivalente o maggiore di salvaguardare la } \\
\text { vita e di ottenere il successo clinico e microbiologico } \\
\text { nei pazienti affetti da gravi infezioni indotte da Gram- } \\
\text { positivi (resistenti) } \\
\text { Possibilità di scelta tra via orale e parenterale }\end{array}$ & $\begin{array}{l}\text { Necessità di associazioni quando la terapia non è mirata } \\
\text { ma emirica } \\
\text { Magiore attenzione ai possibili rischi di mielosoppressione } \\
\text { e di interazione con farmaci }\end{array}$ \\
& $\begin{array}{l}\text { Possibilità di "switch-therapy" iv/os } \\
\text { Possibilità di ridurre la durata della degenza }\end{array}$ & \\
Economico & $\begin{array}{l}\text { Prescrizione costo/efficace (efficiente) quando attuata } \\
\text { secondo indicazioni e linee-guida }\end{array}$ & Costi per eventuali controversie giudiziarie* \\
\end{tabular}

\section{Tabella 6b}

Matrice dei valori per giudicare la convenienza ad usare linezolid nelle polmoniti nosocomiali e comunitarie, nelle infezioni della cute e dei tessuti molli, sostenute da patogeni Gram-positivi resistenti ai comuni antibiotici - Prospettiva del medico curante 


\begin{tabular}{|c|c|c|}
\hline \multicolumn{3}{|c|}{ Prospettiva dell'ospedale } \\
\hline Valore & Positivo & Negativo \\
\hline Clinico & $\begin{array}{l}\text { Probabilità equivalente o maggiore di salvaguardare la } \\
\text { vita e di ottenere il successo clinico e microbiologico } \\
\text { nei pazienti affetti da gravi infezioni indotte da Gram- } \\
\text { positivi (resistenti) } \\
\text { Efficiente controllo delle infezioni nosocomiali } \\
\text { Possibilità di scelta tra via orale e parenterale } \\
\text { Possibilità di "switch-therapy" iv/os } \\
\text { Possibilità di ridurre la durata della degenza }\end{array}$ & $\begin{array}{l}\text { Necessità di associazioni quando la terapia non è mirata } \\
\text { ma empirica } \\
\text { Maggiore attenzione ai possibili rischi di mielosoppressione } \\
\text { e di interazione con farmaci }\end{array}$ \\
\hline Economico & $\begin{array}{l}\text { Riduzione dei costi indotti da infezioni nosocomiali } \\
\text { Risparmio per la possibile riduzione delle giornate di } \\
\text { degenza } \\
\text { Risparmio sui materiali e sul lavoro quando si utilizza } \\
\text { la via orale }\end{array}$ & $\begin{array}{l}\text { Costo di acquisto elevato } \\
\text { Costi per gestire le reazioni avversi } \\
\text { Costi per gestire il rischio di mielosoppressione } \\
\text { Costi per eventuali controversie giudiziarie* }\end{array}$ \\
\hline
\end{tabular}

\section{Tabella 6c}

Matrice dei valori per giudicare la convenienza ad usare linezolid nelle polmoniti nosocomiali e comunitarie, nelle infezioni della cute e dei tessuti molli, sostenute da patogeni Gram-positivi resistenti ai comuni antibiotici - Prospettiva dell'ospedale

\section{Prospettiva del terzo pagante (SSN o assicurazioni)}

\begin{tabular}{|c|c|c|}
\hline Valore & Positivo & Negativo \\
\hline Clinico & $\begin{array}{l}\text { Probabilità equivalente o maggiore di salvaguardare la } \\
\text { vita e di ottenere il successo clinico e microbiologico } \\
\text { nei pazienti affetti da gravi infezioni indotte da Gram- } \\
\text { positivi (resistenti) } \\
\text { Efficiente controllo delle infezioni nosocomiali e } \\
\text { riduzione del rischio di diffusione di ceppi resistenti sul } \\
\text { territorio } \\
\text { Soddisfazione del cliente }\end{array}$ & $\begin{array}{l}\text { Gestione delle reazioni avverse e dei rischi correlati all'uso } \\
\text { extraospedaliero del farmaco }\end{array}$ \\
\hline Economico & $\begin{array}{l}\text { Possibile maggiore efficienza complessiva nell'utilizzo } \\
\text { delle risorse sanitarie con risparmio potenziale sul } \\
\text { costo di gestione di un episodio infettivo in caso di } \\
\text { "switch therapy" e di dimissione precoce del paziente } \\
\text { Possibile risparmio sul costo delle resistenze agli } \\
\text { antibiotici }\end{array}$ & $\begin{array}{l}\text { Parziale spostamento dei costi sanitari dall'ospedale al } \\
\text { territorio in caso di "switch therapy" con dimissione precoce } \\
\text { del paziente }\end{array}$ \\
\hline
\end{tabular}

\section{Tabella 6d}

Matrice dei valori per giudicare la convenienza ad usare linezolid nelle polmoniti nosocomiali e comunitarie, nelle infezioni della cute e dei tessuti molli, sostenute da patogeni Gram-positivi resistenti ai comuni antibiotici - Prospettiva del terzo pagante

\begin{tabular}{lll}
\hline Prospettiva del terzo pagante (SSN o assicurazioni) & \\
\hline Valore & Positivo & Negativo \\
Clinico & $\begin{array}{l}\text { Possibile guadagno di anni di vita Possibile } \\
\text { miglioramento della qualità di vita }\end{array}$ & Indifferente \\
Economico & $\begin{array}{l}\text { Possibile riduzione del costo sociale delle infezioni per } \\
\text { le quali linezolid è indicato }\end{array}$ & Indifferente \\
\hline
\end{tabular}

\section{Tabella 6e}

Matrice dei valori per giudicare la convenienza ad usare linezolid nelle polmoniti nosocomiali e comunitarie, nelle infezioni della cute e dei tessuti molli, sostenute da patogeni Gram-positivi resistenti ai comuni antibiotici - Prospettiva della società

\footnotetext{
* Il costo per eventuali controversie giudiziarie è un costo generico, presente per qualsiasi farmaco e gravante su ospedale o medico
} 


\section{Il costo ospedaliero delle infezioni nosocomiali}

Le infezioni nosocomiali colpiscono mediamente circa l' $8 \%$ dei pazienti ricoverati negli ospedali italiani. Percentuali analoghe, oscillanti tra un ottimale $5 \%$ e un normale $10 \%$, sono state stimate negli ultimi anni nei paesi industrializzati. Con un'elevata frequenza, le infezioni nosocomiali sono indotte da patogeni resistenti o multiresistenti, e in alcuni reparti in particolare da ceppi di MRSA. Le infezioni nosocomiali hanno un elevato impatto sanitario ed economico sull'ospedale e sul SSN e il fenomeno delle resistenze batteriche agli antibiotici è un fattore che incrementa ulteriormente i costi sanitari.

Diversi studi pubblicati negli ultimi anni hanno riportato le stime dei costi aggiuntivi indotti dalle infezioni nosocomiali. Uno dei più completi e interessanti è quello recentemente condotto nel Regno Unito da Plowman e collaboratori, i cui risultati sono parzialmente sintetizzati nelle tabelle 7 e 8 [86]. Lo studio ha monitorato 3.980 pazienti ricoverati, 309 dei quali (7,8\%; CI 95\%: 7,0-8,6\%) hanno sviluppato una o più infezioni acquisite in ospedale.

L'analisi comparativa dei costi indotti sull'ospedale dai pazienti con infezioni nosocomiali, rispetto ai pazienti non infetti, ha evidenziato come l'infezione nosocomiale induca un costo mediamente 2,9 volte superiore, con un incremento equivalente di 3.154 sterline (tabella 7). Anche la degenza media è 2,9 volte maggiore quando il paziente acquisisce l'infezione in ospedale, con un incremento equivalente di oltre 14 giorni. L'incidenza e l'impat- to economico delle infezioni nosocomiali variano sensibilmente in relazione alla sede dell'infezione e al tipo di reparto ospedaliero. Un costo aggiuntivo leggermente inferiore alla media è stato stimato per i pazienti affetti da infezioni polmonari e da infezioni della cute e dei tessuti molli, mentre un costo aggiuntivo più che raddoppiato rispetto alla media è stato stimato per le infezioni multiple.

L'analisi della ridistribuzione degli incrementi tra i vari fattori produttivi dell'assistenza ospedaliera (tabella 8) ha evidenziato come la voce relativa agli antibiotici contribuisca in modo molto modesto $(<2 \%)$ all'incremento totale dei costi correlati al paziente con infezione nosocomiale.

Pertanto, utilizzare farmaci più costosi ma più efficaci per controllare ed eventualmente prevenire i casi di infezioni nosocomiali appare senza dubbio una strategia teoricamente conveniente [86]. Alcuni recenti lavori hanno delineato l'impatto delle infezioni da MRSA sui costi sanitari [87-89]. Secondo gli studi più importanti, l'infezione da MRSA, in confronto con quella da MSSA, induce un costo ospedaliero aggiuntivo variabile da 2.500 a 17.400 dollari [87-89].

Il linezolid è uno dei farmaci più attivi nei confronti dei ceppi MRSA, compresi quelli eventualmente vancomicina-resistenti, e si propone come competitore in alternativa ai glicopeptidi nel trattamento delle infezioni sostenute da MRSA. Analogamente a quanto sopra evidenziato, la quota d'incremento del costo ospedaliero relativa all'uso di antibiotici più costosi, necessari per controllare le gravi

\begin{tabular}{|c|c|c|c|c|c|c|}
\hline & Costo & & & Durata de & la degenza (giorni) & \\
\hline $\begin{array}{l}\text { Sede infezione } \\
\text { nosocomiale }\end{array}$ & $\begin{array}{l}\text { Costo } \\
\text { medio }\end{array}$ & $\begin{array}{l}\text { Rapporto tra } \\
\text { costi* }^{*} \\
\text { (modello: } 95 \% \mathrm{Cl} \text { ) }\end{array}$ & $\begin{array}{l}\text { Costo } \\
\text { aggiuntivo } \\
\text { (modello) }\end{array}$ & $\begin{array}{l}\text { Degenza } \\
\text { media }\end{array}$ & $\begin{array}{l}\text { Rapporto tra giorni } \\
\text { di degenza* } \\
\text { (modello: } 95 \% \mathrm{Cl} \text { ) }\end{array}$ & $\begin{array}{l}\text { Giorni aggiuntivi } \\
\text { (modello) }\end{array}$ \\
\hline $\begin{array}{l}\text { IBVR (Infezioni basse } \\
\text { vie respiratorie) }\end{array}$ & 4027 & $\begin{array}{l}2,5 \\
(2,3: 1,9-2,7)\end{array}$ & $\begin{array}{l}2398 \\
(2080)\end{array}$ & 20,1 & $\begin{array}{l}2,6 \\
(2,1: 1,7-2,6)\end{array}$ & $\begin{array}{l}12,5 \\
(8,4)\end{array}$ \\
\hline Cute e tessuti molli & 3418 & $\begin{array}{l}2,1 \\
(2,1: 1,6-2,5)\end{array}$ & $\begin{array}{l}1790 \\
(1615)\end{array}$ & 19,6 & $\begin{array}{l}2,6 \\
(2,4: 1,8-3,1)\end{array}$ & $\begin{array}{l}12,0 \\
(10,6)\end{array}$ \\
\hline Multiple & 10780 & $\begin{array}{l}6,6 \\
(6,3: 5,4-7,4)\end{array}$ & $\begin{array}{l}9152 \\
(8631)\end{array}$ & 45,4 & $\begin{array}{l}6,0 \\
(4,8: 4,0-5,8)\end{array}$ & $\begin{array}{l}37,8 \\
(29,1)\end{array}$ \\
\hline Tutte le sedi & 4782 & $\begin{array}{l}2,9 \\
(2,8: 2,6-3,0)\end{array}$ & $\begin{array}{l}3154 \\
(2917)\end{array}$ & 21,7 & $\begin{array}{l}2,9 \\
(2,5: 2,3-2,7)\end{array}$ & $\begin{array}{l}14,1 \\
(11,3)\end{array}$ \\
\hline
\end{tabular}

* Rapporto tra costi medi o tra giorni di degenza indotti dal paziente con infezione nosocomiale e indotti dal paziente non infetto

Tabella 7

Impatto dell'infezione nosocomiale sui costi ospedalieri e sulla durata media della degenza, in confronto con pazienti ricoverati non infetti (adattata da: Plowman et al. 2001) [86] 
infezioni nosocomiali da MRSA, è certamente marginale rispetto alle altre voci di costo, prime fra tutte quelle relative alla prolungata permanenza in ospedale [82].

L'uso di linezolid, in virtù della sua elevata efficacia e della sua somministrabilità anche per via orale, può favorire una minore durata della degenza contribuendo a ridurre sensibilmente i costi ospedalieri. La realizzazione di questo obiettivo, tuttavia, non è automatica ed è condizionata da diversi fattori che possono influire sulla dinamica assistenziale e sull'allocazione delle risorse. È stato ipotizzato, ad esempio, che la disponibilità di un farmaco come il linezolid potrebbe comportare la riduzione dei giorni di degenza a fronte di un incremento del costo medio giornaliero dell' assistenza ospedaliera, dovuto ad una gestione più aggressiva del paziente in previsione di dimetterlo precocemente, subito dopo il passaggio dalla via endovenosa al trattamento orale [82].

Inoltre, salvo i casi in cui l'uso di linezolid comporta una riduzione effettiva dei giorni di terapia e di degenza, indipendentemente dall'adozione della "switch-therapy", la dimissione precoce richiede generalmente la continuazione della terapia antibiotica a domicilio, con il conseguente incremento dei costi sanitari a carico del SSN. Si ritiene che tali incrementi siano inferiori ai risparmi indotti a vantaggio dell'ospedale e che, pertanto, la dimissione precoce possa essere comunque conveniente per il SSN. Inoltre, la minor per- manenza in ospedale riduce il disagio che il paziente e i familiari devono affrontare durante il ricovero e quindi contribuisce a migliorare la loro qualità di vita [82, 83].

\section{Costo/efficacia di linezolid versus vancomicina}

Negli ultimi anni sono stati pubblicati tre diversi lavori di farmacoeconomia che hanno valutato la convenienza ad usare il linezolid in sostituzione della vancomicina nel trattamento di infezioni causate da ceppi di stafilococchi per lo più resistenti alla meticillina [81, 83, 84]. Il lavoro più recente analizza la costo/efficacia del linezolid versus vancomicina nel trattamento delle polmoniti nosocomiali da ventilatore (VAP) dovute a Staphylococcus aureus, assumendo la prospettiva del terzo pagante [81]. L'analisi costo/efficacia è stata effettuata mediante un albero decisionale, seguendo i criteri raccomandati per questo tipo di studi dal panel di esperti del National Health Institute (NIH) degli Stati Uniti [90]. Il modello di analisi costo/efficacia incrementale ha considerato come costo la somma dei costi incrementali dovuti all'uso di linezolid e dei costi delle risorse sanitarie consumate dal maggior numero di sopravvissuti, mentre come efficacia sono stati considerati sia gli anni di vita guadagnati dai sopravvissuti nei due gruppi di trattamento, sia gli anni guadagnati ponderati per la qualità di vita (QALYs).

I valori dei parametri di probabilità e di costo del modello sono stati desunti dalla lette-

\begin{tabular}{lll}
\hline Tipo di costo & $\begin{array}{l}\text { Rapporto tra costi del paziente con } \\
\text { infezione acquisita in ospedale e paziente } \\
\text { non infetto }\end{array}$ & $\begin{array}{l}\text { Contributo percentuale al costo } \\
\text { aggiuntivo indotto dall'infezione } \\
\text { nosocomiale }\end{array}$ \\
\hline Spese generali dell'ospedale & 2,5 & 21,43 \\
Direzione amministrativa & 2,3 & 2,07 \\
Costo del capitale & 2,4 & 9,26 \\
Tempo del personale medico & 2,2 & 5,85 \\
Assistenza infermieristica & 4,5 & 42,36 \\
Paramedici e infermieri & 3,6 & 1,47 \\
specializzati & 4,9 & 2,36 \\
Fisioterapia & 1,3 & 2,16 \\
Interventi chirurgici & 11,9 & 3,44 \\
Prodotti monouso (camici, & & 1,83 \\
drenaggi, cannule, ecc.) & 5,3 & 3,47 \\
Antibiotici & 3,7 & 0,83 \\
Tutti gli altri farmaci & 4,8 & 2,06 \\
Esami microbiologici & 2,3 & 0,14 \\
Altri esami di laboratorio & 2,7 & 1,20 \\
Endoscopie & 2,1 & 0,09 \\
Radiologia & 1,5 & 100,00 \\
Altri esami & 2,9 & \\
Totale costi & & \\
&
\end{tabular}

\section{Tabella 8}

Distribuzione tra $i$ vari fattori di costo indicati dell'incremento di costo ospedaliero, indotto da un episodio di infezione nosocomiale (adattata da: Plowman et al. 2001) [86] 


\begin{tabular}{|c|c|c|c|}
\hline Parametri del modello decisionale & Valore base & Range di variazione & Rif. Bibl. \\
\hline Incidenza della VAP da S aureus (\%) & 20,4 & $15,3-25,5$ & 92 \\
\hline Mortalità attribuita a VAP da S aureus (\%) & 27,1 & $20,3-33,9$ & 93 \\
\hline Odds ratio di soprawivenza con linezolid & 1,6 & $1,03-2,40$ & 21 \\
\hline Aspettativa di vita nei soprawissuti (anni) & 9 & $6,75-11,25$ & - \\
\hline Fattore di conversione QALY & 0,83 & $0,62-1,00$ & 41 \\
\hline Costo incrementale linezolid per ciclo terapeutico (\$) & 1,000 & $750-1.250$ & - \\
\hline Costo ospedaliero per VAP $(\$)$ & 12.432 & $9.324-15.540$ & 94 \\
\hline Costi sanitari annuali post-ricovero $(\$)$ & 16.446 & $12.335-20.558$ & 41 \\
\hline Tasso di sconto annuale (\%) & 3 & $0-6$ & - \\
\hline
\end{tabular}

\section{Tabella 9}

Parametri del modello decisionale analisi costo/efficacia del linezolid vs vancomicina nel trattamento della VAP da S. aureus: valori assunti nello scenario base e range di variazione usati nell'analisi di sensibilità (da: Shorr AF et al, 2004) [81]

ratura e i costi, valorizzati al 2001, rispecchiano le condizioni del sistema sanitario statunitense. Il guadagno in sopravvivenza attribuito al linezolid (1,6 volte la vancomicina) è stato ricavato dai risultati dello studio di Kollef e collaboratori, sopra citato [21]. La tabella 9 riporta il valore dei parametri assunti nel modello per lo scenario base e per l'analisi di sensibilità. La tabella 10 riassume i risultati dell'analisi.

Il linezolid è significativamente più costoso della vancomicina, ma i risultati dello studio di Kollef e collaboratori indicano che il linezolid è anche più efficace della vancomicina nel trattamento delle VAP da S. aureus, soprattutto quando si tratta di MRSA.

La maggior efficacia si manifesta non solo con una maggiore frequenza di pazienti curati, ma anche con un maggior numero di sopravvissuti e quindi con un guadagno in anni di vita e in QALYs [21]. L'analisi costo/efficacia incrementale condotta secondo lo scenario base e i costi statunitensi indica che il costo per ogni soggetto in più salvato dalla morte è di oltre 67.000\$, con possibili oscillazioni entro un range di valori molto ampio (21.243\$ $321.000 \$)$. Il costo per anno di vita guadagnato è stato stimato in $22.072 \$$, con oscillazioni possibili tra $10.669 \$$ e $66.367 \$$, mentre il costo per QALY aggiuntivo è stato stimato in 29.945, con oscillazioni possibili tra $13.324 \$$ e $101.143 \$$.

Questi valori della costo/efficacia incrementale rientrano entro i limiti convenzionalmente assunti come accettabili per i paesi industrializzati (approssimativamente 100.000\$/QALY).

Il modello è moderatamente sensibile ai livelli di efficacia incrementale del linezolid sulla vancomicina. Tuttavia, anche nel caso peggiore in cui tutti i parametri assumano il valore più sfavorevole, il linezolid rimane una opzione costo/efficace.Le conclusione degli autori sono che il linezolid rappresenta un'alternativa costo/efficace, cioè conveniente, rispetto alla vancomicina nel trattamento della polmonite nosocomiale da ventilatore (VAP) [81].

Il vantaggio economico del linezolid sulla vancomicina si evince chiaramente anche dal lavoro clinico di Li e collaboratori, sopra esaminato [65]. La disponibilità di linezolid orale agevola l'adozione di strategie sequenziali con switch iv/os e conseguente possibile riduzione dei gironi di degenza in ospedale. In questo lavoro non è stato affrontato esplicitamente, in chiave analitica, il tema del diverso consumo di risorse sanitarie correlabile alla riduzione delle giornate di degenza. Tuttavia, i risultati clinici di questo studio sono stati ripresi e commentati in chiave farmacoeconomica da Dilip Nathwani [82].

\begin{tabular}{lrr}
\hline Parametro C/E incrementale del linezolid & Scenario base del modello & Analisi sensibilità multivariata \\
\hline & $\begin{array}{l}\text { Scenario del } \\
\text { caso peggiore }\end{array}$ & $\begin{array}{l}\text { Scenario del } \\
\text { caso migliore }\end{array}$ \\
Costo incrementale per incremento di un sopravvissuto & $\$ 67.202$ & $\$ 320.958$ \\
Costo incrementale per incremento di un anno di vita guadagnato & $\$ 22.072$ & $\$ 66.367$ \\
Costo incrementale per incremento di un QALY & $\$ 29.945$ & $\$ 10.659$ \\
\hline
\end{tabular}

\section{Tabella 10}

Risultati di un'analisi costo/efficacia incrementale attuata con un modello decisionale ad albero per confrontare la convenienza del linezolid vs vancomicina nel trattamento dei pazienti affetti da polmonite associata a ventilatore (VAP) sostenuta da Staphylococcus aureus (da: Shorr AF et al. 2004) [81] 
I risultati clinici e gli outcomes sanitari dello studio di Li e collaboratori sono stati utilizzati anche in una recente analisi farmacoeconomica condotta da Ravasio e tarata sui costi ospedalieri italiani [83]. I risultati sono riportati nella tabella 11 .

Secondo questa valutazione, la scelta del linezolid può comportare un sensibile risparmio per l'ospedale e per il SSN, qualora sia possibile ridurre le giornate di degenza, grazie alla "switch-therapy", e continuare la terapia orale con linezolid a domicilio. La riduzione delle giornate di degenza comporta un risparmio medio per l'ospedale stimato in oltre 2.600 euro per paziente, mentre il trattamento domiciliare induce un maggior costo stimato in 1.135 euro. Il risparmio netto medio per il SSN potrebbe essere di circa 1.500 euro per paziente trattato con linezolid iv/os in alternativa alla vancomicina iv [83].

Un gruppo di ricercatori statunitensi hanno valutato, mediante un adatto modello decisionale ad albero, il possibile impatto farmacoeconomico derivante dall'adottare, nei pazienti affetti da infezioni sostenute da MRSS, un protocollo sequenziale che prevede di passare dal trattamento endovenoso con vancomicina al trattamento orale con linezolid e quindi dimettere precocemente questi pazienti, quando le condizioni cliniche lo consentono [84]. In questo studio gli autori hanno riesaminato retrospettivamente le cartelle cliniche dei pazienti ricoverati nel 2000 presso il Veterans Affaire Greater Los Angeles Healthcare System Hospital e trattati per almeno 7 giorni con vancomicina per sospetta o accertata infezione da MRSS.

Sono stati identificati complessivamente 177 pazienti, dei quali 74 (42\%) sono stati giudicati non eligibili per la "switch-therapy" e la dimissione precoce, mentre $103(58 \%)$ sono stati considerati eligibili per il passaggio dalla

\begin{tabular}{lll}
\hline Costi di trattamento (in euro) & $\begin{array}{l}\text { Linezolid } \\
(\mathbf{n}=\mathbf{1 2 4})\end{array}$ & $\begin{array}{l}\text { Vancomicina } \\
(\mathbf{n}=\mathbf{1 3 0})\end{array}$ \\
\hline Trattamento ospedaliero & $4.002,14$ & $6.607,44$ \\
$\quad$ Degenza & $3.104,54$ & $6.300,39$ \\
$\quad$ Antibiotico & 897,60 & 307,05 \\
$\quad$ Trattamento domiciliare & $1.135,20$ & 0 \\
Costo Totale & $5.137,34$ & $6.607,44$ \\
Differenziale & & $+1.470,10$ \\
\hline
\end{tabular}

\section{Tabella 11}

Costi di trattamento stimati per l'Italia sulla base dei risultati dello studio internazionale multicentrico di Fase III* (modificato da: Ravasio, 2003) [83]

* Li Z. et al, 2003 [65] vancomicina iv al linezolid orale. Inoltre, $55 \mathrm{di}$ questi 103 pazienti (31\% del campione totale) sono stati giudicati eligibili per la dimissione precoce a domicilio, mentre i rimanenti 48 non lo sono stati. I criteri di eligibilità per la "switchtherapy" e la dimissione precoce sono stati quelli proposti da Ramirez e collaboratori [91]. Per i pazienti giudicati dimissibili precocemente, le condizioni per realizzare la switch dalla vancomicina iv al linezolid orale si realizzavano mediamente $3,3 \pm 2,9$ giorni prima della reale data di dimissione.

Gli autori hanno stimato che l'ospedale avrebbe risparmiato ben $294.750 \$$ (range $35.730 \$-553.790 \$$ ), qualora i 55 pazienti eligibili avessero realizzato la "switch-therapy" e fossero stati dimessi precocemente. Al contrario, la conversione da vancomicina iv, meno costosa, al linezolid orale, più costoso, dei 48 pazienti eligibili per la "switch-therapy" ma non dimissibili precocemente, avrebbe comportato per l'ospedale un incremento di costi molto contenuto, stimato in $6.340 \$$ (range variabile da -12.910\$ a 11.900) [84]. Secondo gli autori, l'analisi modellistica condotta da loro dimostra come sia conveniente scegliere di utilizzare il linezolid orale nei pazienti affetti da infezioni da MRSS inizialmente trattati con vancomicina endovenosa e convertibili alla via orale.

L'incremento dei costi di acquisto degli antibiotici, dovuto al maggiore costo del linezolid, viene ampiamente neutralizzato dai maggiori risparmi ottenibili con la dimissione precoce e il risparmio di giornate di degenza molto costose [84].

\section{Costo/efficacia di linezolid versus teicoplanina}

Un gruppo di ricercatori ha valutato comparativamente il consumo di risorse ospedaliere e i relativi costi associati all'uso di linezolid, in protocolli di "switch-therapy" iv/os di pazienti affetti da gravi infezioni batteriche da Grampositivi, in confronto con la teicoplanina usata per via parenterale, solo per via endovenosa o per via endovenosa con successiva conversione alla via intramuscolare [85]. L'analisi è stata condotta nel corso di una ricerca clinica di Fase IIIb, multicentrica, internazionale, condotta in 6 diversi centri del Sud America (Argentina, Cile, Columbia e Venezuela) e del Messico. Il protocollo sperimentale era identico a quello sopra discusso [67]. Un totale di 203 pazienti sono stati inclusi nell'analisi farmacoeconomica, 97 trattati con linezolid e 106 con teicoplanina. Il 58,6\% era affetto da infezioni della cute e dei tessuti molli, il 36,5\% da polmonite e il $3 \%$ da batteriemia. 
La percentuale di successi alla visita di fine trattamento è risultata equivalente nei due gruppi: 98,9\% nel gruppo linezolid e 93,9\% nel gruppo teicoplanina. La mortalità, invece è stata significativamente superiore nel gruppo teicoplanina $(9,4 \%$ contro il $2,1 \%$ del linezolid, $\mathrm{p}=0,03$ ) [85]. La durata della degenza è stata inferiore nel gruppo linezolid rispetto al gruppo teicoplanina, come riportano la tabella $12 \mathrm{e}$ la figura 6. I pazienti trattati con linezolid hanno consumato mediamente 2,2 gironi di degenza in meno; la mediana della degenza è di 2 giorni inferiore nel gruppo linezolid.

La frequenza delle dimissione nel gruppo linezolid è stata più elevata che nel gruppo teicoplanina durante le prime tre settimane di terapia.

La terapia per via endovenosa è stata mediamente 3,1 giorni più breve nel gruppo linezolid $(\mathrm{p}<0,001)$ rispetto al gruppo teicoplanina, mentre la durata complessiva della terapia (iv + os nel caso del linezolid e iv + im nel caso della teicoplanina) non è risultata statisticamente differente nei due gruppi di trattamento (tabella 12).

Il costo medio totale per paziente è stato stimato in $3.099 \$$ per il gruppo linezolid e in $3.410 \$$ per il gruppo teicoplanina; una differenza che non è statisticamente significativa. L'analisi dei fattori di costo indica che la scelta del linezolid comporta un incremento medio per paziente di $215 \$$, statisticamente significativo rispetto al costo della teicoplanina. Tuttavia, l'uso di linezolid induce un risparmio su tutte le altri voci di costo, e in particolare un risparmio medio di $444 \$$ per paziente grazie alla riduzione delle giornate di degenza.

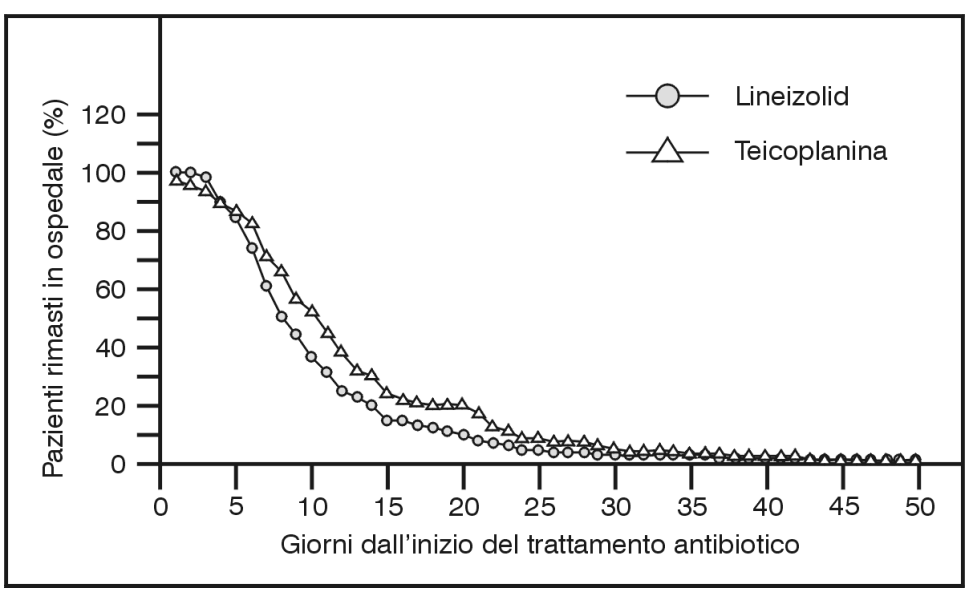

Figura 6

Curve di Kaplan-Meier che rappresentano la probabilità dei pazienti affetti da gravi infezioni sostenute da Gram-positivi di rimanere ricoverati a partire dall'inizio del trattamento antibiotico con linezolid o con teicoplanina (modificata da: Lopez H et al, 2003) [85]

Il bilancio finale, seppure non statisticamente significativo, registra un risparmio netto per paziente di $311 \$$ quando si sceglie di usare linezolid in alternativa alla teicoplanina [85].

\section{CONCLUSIONI}

Linezolid, il primo antibatterico di sintesi della nuova classe degli oxazolidinoni entrato nell'uso clinico, rappresenta un importante e prezioso strumento per curare le infezioni polmonari, nosocomiali e comunitarie, e le infezioni complicate della cute e dei tessuti molli, causate da patogeni Gram-positivi, compresi gli stafilococchi ed enterococchi meticillino-e

\begin{tabular}{|c|c|c|c|c|c|c|}
\hline & \multicolumn{3}{|c|}{ Inclusi i pazienti deceduti } & \multicolumn{3}{|c|}{ Esclusi i pazienti deceduti } \\
\hline & LIN & TEICO & $\mathbf{P}$ & LIN & TEICO & $P$ \\
\hline$N^{\circ}$ pazienti & 97 & 106 & & 95 & 96 & 0 \\
\hline No morti (\%) & - & - & & $2(2,1 \%)$ & $10(9,4 \%)$ & 0,026 \\
\hline Giorni degenza (KM) & & & 0,030 & & & 0,055 \\
\hline Media & 9,8 & 12,0 & & 9,8 & 11,8 & \\
\hline Mediana & 8 & 10 & & 8 & 9 & \\
\hline $1^{\circ}$ quartile & 5 & 6 & & 5 & 6 & \\
\hline $3^{\circ}$ quartile & 12 & 14 & & 11 & 14 & \\
\hline \multicolumn{7}{|c|}{$\begin{array}{l}\text { Giorni di terapia antibiotica } \\
\text { Media }\end{array}$} \\
\hline Solo endovena & 6,1 & 9,2 & $<0,001$ & 6,1 & 9,3 & $<0,001$ \\
\hline Totale (iv+os/im) & 14,2 & 13,5 & 0,418 & 14,3 & 13,9 & 0,651 \\
\hline
\end{tabular}

\section{Tabella 12}

Giorni di degenza e durata media (giorni) del trattamento antibiotico con linezolid o teicoplanina in un gruppo di pazienti affetti da gravi infezioni sostenute da Gram-positivi (dati da: Lopez H et al, 2003) [85] 
vancomicina-resistenti, gli pneumococchi penicillino-resistenti ed eritromicina-resistenti.

Il linezolid si presenta, dunque come il naturale e più accreditato competitore dei glicopeptidi, vancomicina e teicoplanina, per queste indicazioni.

Il profilo di efficacia nelle polmoniti nosocomiali (comprese le VAP) e comunitarie, nelle infezioni complicate della cute e dei tessuti molli e nei casi di batteriemia è stato documentato in diversi studi controllati, multicentrici e internazionali.

Durante gli studi clinici e successivamente nella fase di post-marketing è stato delineato un profilo di sicurezza e tollerabilità equivalente a quello dei farmaci coi quali è stato confrontato.

Il rapido assorbimento del linezolid dal tratto gastrointestinale, la sua biodisponibilità orale pressoché totale e la disponibilità in commercio di una formulazione orale del prodotto, a fianco della tradizionale formulazione endovenosa, sono caratteristiche che consentono di effettuare strategie sequenziali e la conversione di molti pazienti dalla via endovenosa alla via orale, non appena le condizioni cliniche del paziente si sono stabilizzate. L'uso orale del farmaco facilita la dimissione precoce dall'ospedale e la prosecuzione della terapia antibiotica a domicilio.

Studi clinici controllati e verifiche a posteriori hanno documentato che questo risultato si realizza e può essere raggiunto, con interessanti risparmi per l'ospedale e per il SSN.

In particolare il linezolid è risultato costo/ efficace, cioè conveniente, in alternativa alla vancomicina e alla teicoplanina nel trattamento delle polmoniti nosocomiali e nelle infezioni gravi causate da stafilococchi meticillino-resistenti.

\section{BIBLIOGRAFIA}

1. Jones RN, Low DE, Pfaller MA. Epidemiologic trends in nosocomial and community-acquired infections due to antibiotic-resistant Gram-positive bacteria: the role of streptogramins and other newer compounds. Diagn Microb Infcet Dis 33: 101-112, 1990.

2. Maschmeyer $\mathrm{G}$ et al. Changing patterns of infections and antimicrobial susceptibilities. Oncology 14 (Suppl 6): 9$16,2000$.

3. Oppenheim BA. The changing pattern of infection in neutropenic patients. J Antimicrob Chemother 41(SupplD): 711,1998 .

4. Voss A et al. Methicillin-resistant Staphylococcus aureus in Europe. Eur J Clin Microbiol Infect Dis 13 : 50-55, 1994.

5. Gold HS, Moellering RCJr. Antimicrobial-drug resistance. N Engl J Med 335: 1445-1453, 1996.

6. Pfaller et al. Bacterial pathogens isolated from patients with bloodstream infections: frequencies of occurrence and antimicrobial susceptibility patterns from the SENTRY Antimicrobial Surveillance Program. Antimicrob Agents Chemother 42: 1762-1770, 1998.

7. Fridkin SK et al. Antimicrobial resistance in Intensive Care Units. Clin Chest Med 20: 303-316, 1999.

8. Marchese A et al. Emergence of drug-resistant Gram-positive pathogenic bacteria. J Chemother 12 (Suppl2): 12-14, 2000.

9. Moellering RC. Quinupristin/dalfopristin: therapeutic potential for vancomycin-resistant enterococcal infections. J Antimicrob Chemother 44 (Suppl A): 25-30, 1999.

10. Livermore DM. Quinupristin/dalfospristin and linezolid: where, when, which and whether to use? J Antimicrob Chemother 46: 347-350, 2000.

11. Moellering RC Jr. Linezolid: the first oxazolidinone antimicrobial. Ann Intern Med 138: 135-142, 2003

12. Brickner SJ. Oxazolidinone antibacterial agents. Current Pharmaceutical Design 2: 175-194, 1996.

13. Ford C, Hamel J, Stapert D, et al. Oxazolidinones: a new class of antimicrobials. Infect in Med 16: 435-445, 1999.

14. Zyvoxid-Scheda tecnica di registrazione in Italia, rev. novembre 2002, Pharmacia Italia SpA, ora Pfizer Italia SpA.

15. Clemett D, Markham A. Linezolid. Drugs 59:815-827, 2000.

16. Diekema DJ \& Jones RN. Oxazolidinones - a review. Drugs 59:7-16, 2000. 
17. Perry CM, Jarvis B. Linezolid: a review of its use in the management of serious gram-positive infections. Drugs 61: 525-551, 2001.

18. Corti G, Cinelli R, Paradisi F. Clinical and microbiological efficacy and safety profile of linezolid, a new oxazolidinone antibiotic. Int J Antimicrob Agents 16: 527-530, 2000.

19. Stevens DL, Herr D, Lampiris H, et al. Linezolid versus vancomycin for the treatment of methicillin-resistant Staphylococcus aureus infections. Clin Infect Dis 34: 1481-1490, 2002.

20. Wunderink RG, Rello J, Cammarata SK, et al. Linezolid vs vancomycin: Analysis of two double-blind studies of patients with methicillin-resistant Staphylococcus aureus nosocomial pneumonia. Chest 124: 1789-1797, 2003.

21. Kollef MH, Rello J, Cammarata SK, et al. Clinical cure and survival in Gram-positive ventilator-associated pneumonia: retrospective analysis of two double-blind studies comparing linezolid with vancomycin. Int Care Med 30: 388-394, 2004.

22. Wilcox M, Tang T, Hafkin B. Linezolid versus teicoplanin for the treatment of hospitalized patients with grampositive infections. In: Program and Abstracts of the 41 Annual Interscience Conference on Antimicrobial Agents and Chemotherapy, September 22, 2001, Chicago, Illinois. Washington, DC: American Society for Microbiology, Abstract 1481, 2001.

23. Jones RN, Johnson DM \& Erwin ME. In vitro spectrum and activity of U-100592 and U-100766, two novel oxazolidinones. Presented at the 35th Interscience Conference on Antimicrobial Agents and Chemotherapy; San Diego, CA, USA, Sept 17, 1995.

24. Eliopoulos GM, Wennersten CB, Gold HS et al. In vitro activities of new oxazolidinone antimicrobial agents against enterococci. Antimicrob Agents Chemother 40:1745-1747, 1996.

25. Mason Jr EO, Lamberth LB \& Kaplan SL. In vitro activities of oxazolidinones U-100592 and U-100766 against penicillin-resistant and cephalosporin-resistant strains of Streptococcus pneumoniae. Antimicrob Agents Chemother 40:1039-1040, 1996.

26. Zurenko GE, Yagi BH, Schaadt RD, et al. In vitro activities of U-100592 and U-100766, novel oxazolidinone antibacterial agents. Antimicrob Agents Chemother 40: 839-845, 1996.

27. Genin MJ, Allwine DA, Hutchinson DK et al. Substituent effects on the antibacterial activity of novel highly potent nitrogen-bound azolylphenyl oxazolidinones. Presented at the 38th Interscience Conference on Antimicrobial Agents and Chemotherapy; San Diego, CA, USA, Sept 24-27, 1998. Goldstein EJC, Citron SH, Gerardo CV: Linezolid compared with other macrolides against Pasteurella species. Presented at the 38th Interscience Conference on Antimicrobial Agents and Chemotherapy; San Diego, CA, USA, Sept 24-27, 1998.

28. Piper KE, Rouse MS, Patel R et al. In-vitro activity of linezolid against vancomycin-and mechanism of resistance. Int J Antimicrob Agents 23: 113-119, 2004.

29. Verbist L \& Verhaegen J. In-vitro activity of linezolid against gram-positive cocci. Presented at the 38th Interscience Conference on Antimicrobial Agents and Chemotherapy; San Diego, CA, USA, Sept 24-27, 1998.

30. Wu M, Aralor P, Nash K et al. Linezolid, a new oxazolidinone, has activity in vitro and in macrophage culture system against mycobacterium avium complex (MAC). Presented at the 38 th Interscience Conference on Antimicrobial Agents and Chemotherapy; San Diego, CA, USA, Sept 24-27, 1998.

31. Henwood CJ, Livermore DM, Johnson AP, et al. Susceptibility of gram-positive cocci from 25 UK hospitals to antimicrobial agents including linezolid. The Linezolid Study Group. J Antimicrob Chemother 46: 931-940, 2000.

32. Stefani $\mathrm{S}$ et al. Attività antibatterica in vitro di linezolid nei confronti di stafilococchi ed enterococchi isolati in Italia. GIM-MOC, Quaderni di Microbiologia Clinica 5 (V):1-10, 2001.

33. Cercenado E, Garcia.Garrote F, Bouza E. In vitro activity against multiply resistant Gram-positive clinical isolates. J Antimicrob Chemother 47: 77-81, 2001.

34. Ford CW, Hamel JC, Moerman JK et al. In vivo efficacy evaluations of U-100592 and U-100766, new oxazolidinone antibiotics. Presented at the 35th Interscience Conference on Antimicrobial Agents and Chemotherapy; San Diego, CA, USA, Sept 17, 1995.

35. Ford CW, Hamel JC, Wilson DM et al. In vivo activities of U-100592 and U-100766, novel oxazolidinone antimicrobial agents, against experimental bacterial infections. Presented at the 38th Interscience Conference on Antimicrobial Agents and Chemotherapy; San Diego, CA, USA, Sept 24-27, 1998.

36. Andes D, Van Ogtrop ML \& Craig WA. Pharmacodynamic activity of a new oxazolidinone, linezolid, in an animal infection model. Presented at the 38th Interscience Conference on Antimicrobial Agents and Chemotherapy; San Diego, CA, USA, Sept 24-27, 1998. 
37. Swaney SM, Aoki H, Ganoza MC et al. The oxazolidinone linezolid inhibits initiation of protein synthesis in bacteria. Antimicrob Agents Chemother 42:3251-3255, 1998.

38. Lin AH, Murray RW, Vidmar TJ, et al. The oxazolidinone eperezolid binds to the 50S ribosomal subunit and competes with binding of chloramphenicol and lincomycin. Antimicrob Agents Chemother 41: 2127-31, 1997.

39. Bozdogan B, Appelbaum PC. Oxazolidinones: activity, mode of action, and mechanism of resistance. Int J Antimicrob Agents 23: 113-119, 2004.

40. Gonzales RD, Schreckenberger PC, Graham MB et al. Infections due to vancomycin-resistant Enterococcus faecium resistant to linezolid. Lancet 357:1179, 2001.

41. Herrero IA, Issa NC, Patel R. Nosocomial spread of linezolid-resistant, vancomycin-resistant Enterococcus faecium. N Engl J Med 346:867-869, 2002.

42. Tsiodras S, Gold HS, Sakoulas G et al. Linezolid resistance in a clinical isolate of Staphylococcus aureus. Lancet 358:207-208, 2001.

43. Hyatt JM, Ballow CH, Forrest A et al. Safety and efficacy of linezolid (PNU-100766) in eradication of nasal Staphylococcus aureus. Presented at the 38th Interscience Conference on Antimicrobial Agents and Chemotherapy; San Diego, CA, USA, Sept 24-27, 1998.

44. Rayner CR, Forrest A, Meagher AKm et al. Clinical pharmacodynamics of linezolid in seriously ill patients treated in a compassionate use programme. Clin Pharmacokinet 42: 1411-1423, 2003.

45. Stalker DJ, Jungbluth GL. Clinical pharmacokinetics of linezolid, a novel oxazolidinone antibacterial. Clin Pharmacokinet 42: 1129-1140, 2003.

46. Stalker DJ, Jungbluth GL, Hopkins NK, et al. Pharmacokinetics and tolerance of single-and multiple-dose oral or intravenous linezolid, an oxazolidinone antibiotic, in healthy volunteers. J Antimicrob Chemother 51: 1239-1246, 2003.

47. Pawsey SD, Daley-Yates PT, Wajszczuk CP et al. U-100766 safety, toleration and pharmacokinetics after oral and intravenous administration (abstract). First European Congress of Chemotherapy: May 14, 1996; F151. Presented at the 38th Interscience Conference on Antimicrobial Agents and Chemotherapy; San Diego, CA, USA, Sept 24-27, 1996.

48. Turnak MR, Forrest A, Hyatt JM et al. Multiple-dose pharmacokinetics of linezolid-200, 400, and $600 \mathrm{mg} P O Q 12$ $h$. Presented at the 38th Interscience Conference on Antimicrobial Agents and Chemotherapy; San Diego, CA, USA, Sept 24-27, 1998.

49. Kearns GL, Abdel-Rahman SM, Blumer JL et al. Single dose pharmacokinetics of linezolid in infants and children. Pediatr Infect Dis J 19:1178-1184, 2000.

50. Shaikh ZHA, Peloquin CA, Ericsson CD. Successful treatment of vancomycin-resistant Enterococcus faecium meningitis with linezolid: case report and literature review. Scand J Infect Dis 33:375-379, 2001.

51. Kaplan SL, Patterson L, Edwards KM et al. Linezolid for the treatment of community-acquired pneumonia in hospitalized children. Pediatr Infect Dis J 20:488-494, 2001.

52. Rao GG, Steger A, Tobin CM. Linezolid levels in pancreatic secretions. J Antimicrob Chemother 48:931-932, 2001.

53. Feenstra KL, Slatter JG, Stalker DJ et al. Metabolism and excretion of the oxazolidinone antibiotic linezolid (PNU100766) following oral administration of (C)PNU-100766 to healthy volunteers. Presented at the 38th Interscience Conference on Antimicrobial Agents and Chemotherapy; San Diego, CA, USA, Sept 24-27, 1998.

54. Chiba K, Feenstra KL, Slater JG et al. Absorption, distribution, metabolism, and excretion of the oxazolidinone antibiotic linezolid (PNU-100766) in the Sprague Dawley rat. Presented at the 38th Interscience Conference on Antimicrobial Agents and Chemotherapy; San Diego, CA, USA, Sept 24-27, 1998.

55. Brier ME, Stalker DJ, Aronoff GR et al. Pharmacokinetics of linezolid in subjects with varying degrees of renal function and on suspected or proven Gram-positive infections. J Antimicrob Chemother 53: 335-344, 2004.

56. Bosso JA, Flume PA, Gray SL. Linezolid pharmacokinetics in adults patients with cystic fibrosis. Antimicrob Agents Chemother 48: 281-284, 2004.

57. Meagher AK, Forrest A, Rayner CR, et al. Population pharmacokinetics of linezolid in patients treated in a compassionate-use program. Antimicrob Agents Chemother 47: 548-553, 2003.

58. Plouffe JF. Emerging therapies for serious gram-positive bacterila infections: a focus on linezolid. Clin Infect Dis 31 (Suppl 4): S144-149, 2000. 
59. Stevens DL, Smith LG, Bruss JB, et al. Randomized comparison of linezolid (PNU-100766) versus oxacillindicloxacillin for treatment of complicated skin and soft tissue infections. Antimicrob Agents Chemother 44: 34083413, 2000.

60. Vinken AG, Li JZ, Balan DA, et al. Comparison of linezolid with oxacillin or vancomycin in the empiric treatment of cellulitis in US hospitals. Am J Ther 10: 264-274, 2003.

61. Cammarata SK, Schueman LK, Timm JA, et al. Oral linezolid in the treatment od community-acquired pneumonia: a phase III trial. In: Program and abstracts of the American Thoracic Society Annual Meeting, 2000 (Toronto). New York: American Thoracic Society, Bastrac E73, pag 654, 2000.

62. Cammarata SK, Bermudez M, Golin V, et al. Comparison of linezolid versus ceftriaxone/cefpodoxime in the treatment of hospitalized community-acquired pneumonia. In: Program and Abstracts of the 9th International Congress on Infectious Diseases; April 10, 2000; Buenos Aires, Argentina. Boston, Mass: International Society of Infectious Diseases, Abstract 80.008, pag 181, 2000.

63. Rubinstein E, Cammarata SK, Oliphant TH et al. Linezolid (PNU-100766) versus vancomycin in the treatment of hospitalized patients with nosocomial pneumonia: a randomized, double-blind, multi-center study. Clin Infect Dis 32:402-412, 2001.

64. Wunderink RG, Cammarata SK, Oliphant TH, et al. Continuation of a randomized, double-blind, multicenter study of linezolid versus vancomycin in the treatment of patients with nosocomial pneumonia. Clin Ther 25: 980-992, 2003.

65. Li Z, Willke RJ, Pinto LA, et a. Comparison of length of hospital stay for patients with known or suspected methicillin-resistant Staphylococcus species infections treated with linezolid or vancomycin: a randomized, multicenter trial. Pharmacotherapy 21: 263-274, 2001.

66. Wilcox M, Nathwani D, Dryden M. Linezolid compared with teicoplanin for the treatment of suspected or proven Gram-positive infections. J Antimicrob Chemother 53: 335-344, 2004.

67. Cepeda JA, Whitehouse T, Cooper B, et al. Linezolid versus teicoplanin in the treatment of Gram-positive infections in the critically ill: a randomized, double-blind, multicentre study. J Antimicrob Chemother 53: 345-355, 2004.

68. Rubinstein E, Isturiz R, Standiford HC, et al. Worldwide assessment of linezolid's clinical safety and tolerability: comparator-controlled Phase III studies. Antimicrob Agents Chemother 47: 1824-1831, 2003.

69. Bernsein WB, Trotta RF, Rector JT et al. Mechanisms for linezolid-induced anemia and thrombocytopenia. Ann Pharmacother 37:517-520, 2003.

70. Green SL, Maddox JC \& Huttenbach ED. Linezolid and reversible myelosuppression. JAMA 285:1291, 2001.

71. Halpern M. Linezolid-induced pancytopenia. Clin Infect Dis 35:347-348, 2002.

72. Monson T, Schichman SA \& Zent CS. Linezolid-induced pure red blood cell aplasia. Clin Infect Dis 35:e29-e31, 2002.

73. Orrick JJ, Johns T, Janelle J et al. Thrombocytopenia secondary to linezolid administration: What is the risk? Clin Infect Dis 35:348-349, 2002.

74. Waldrep TW \& Skiest DJ. Linezolid-induced anemia and thrombocytopenia. Pharmacotherapy 22:109-112, 2002.

75. Humphrey SJ, Curry JT, Turman CN, et al. Cardiovascular sympathomimetic amine interactions in rats treated with mono-amine oxidase inhibitors and the novel oxazolidinone antibiotic linezolid. J Cardiovasc Pharmacol 37: 548$563,2001$.

76. Hendershot P, Antal E, Welshman I et al. Linezolid: pharmacokinetic and pharmacodynamic evaluation of coadministration with pseudoephedrine $\mathrm{HCl}$, phenylpropanolamine $\mathrm{HCl}$, and dextromethorphan $\mathrm{HBr}$. J Clin Pharmacol 41:563-572, 2001.

77. Sternbach H. The serotonin syndrome. Am J Psychiatry 148:705-713, 1991.

78. Lavery S, Ravi RH, McDaniel WW, et al. Linezolid and serotonin syndrome. Psychosomatics 42: 432-434, 2001.

79. Wigen TW, Goetz MB. Serotonin syndrome and linezolid. Clin Infect Dis 34: 1651-1652, 2002.

80. Kahneman D, Twersky A. Choices, values, and frames. Am Psychologist 39: 341-450, 1984.

81. Shorr AF, Susla GM, Kollef MH. Linezolid for treatment of ventilator-associated pneumonia: a cost-effective alternative to vamcomycin. Crit Care Med 32: 137-143, 2004.

82. Nathwani D. Impact of methicillin-resistant Staphilococcus aureus infections on key health economic outcomes: does reducing the length of hospital stay matter? J Antimicrob Chemoher 51(S2): 37-44, 2003. 
83. Ravasio R. I costi della degenza ospedaliera di pazienti affetti da infezioni da Staphylococcus aureus meticillinoresistenti (MRSA) trattati con linezolid o vancomicina. PharmaHospital, 2003.

84. McCollum M, Rhew DC, Parodi S. Cost analysis of switching from iv vancomycin to PO linezolid for the management of methicillin-resistant Staphylococcus aureus species. Clin Ther 25: 3173-3189, 2003.

85. Lopez H, Li JZ, Balan DA, et al. Hospital resource use and cost of treatment with linezolid versus teicoplanin for tretament of serious Gram-positive bacterial infections among hospitalized patients from South America and Mexico: results from a multicenter trial. Clin Ther 25: 1846-1871, 2003.

86. Plowman R, Graves N, Griffin MAS, et al. The rate and cost of hospital-acquired infections occurring in patients admitted to selected specialties of a district general hospital in England and the national burden imposed. $\mathrm{J}$ Hosp Infect 47: 198-209, 2001

87. Rubin RJ Harrington CA, Poon A, et al. The economic impact of Staphylococcus aureus infection in New York City hospitals. Emerging Infect Dis 5: 9-17, 1999.

88. Abramson MA, Sexton DJ. Nosocomial methicillin resistant and methicillin susceptible Staphylococcus aureus primary bacteraemia: at what costs? Inf Control Hosp Epidemiol 20: 408-411, 1999.

89. Kim T, Oh PI, Simor AE. The economic impact of methicillin-resistant Staphylococcus aureus in Canadian hospitals. Inf Control Hosp Epidemiol 22: 99-104, 2001.

90. Weinstein MC, Siegel JE, Gold MR, et al. Recommendations of the Panel on Cost-effectiveness in Health and Medicine. JAMA 276: 1253-1258, 1996.

91. Ramirez JA, Vargas S, Ritter GW, et al. Early switch from intravenous to oral antibiotics and hospital discharge: a prospective observational study of 200 consecutive patients with community-acquired pneumonia. Arch Intern Med 159: 2449-2454, 1999.

92. Quartin AA; Schein RM, Kett DH, et al. Magnitude and duration of the effect of sepsis on survival. JAMA 277: 1058$1063,1997$.

93. Angus DC, Linde-Zwirble WT, Clermont G, et al. Cost-effectiveness of drotregogin alfa (activated) in the treatment of severe sepsis. Crit Care Med 31: 1-11, 2003.

94. Warren DK, Shukla SJ, Olsen MA, et al. Outcome and attributable cost of ventilator-associated pneumonia among intensive care unit patients in a suburban medical center. Crit Care Med 31: 1312-1317, 2003. 